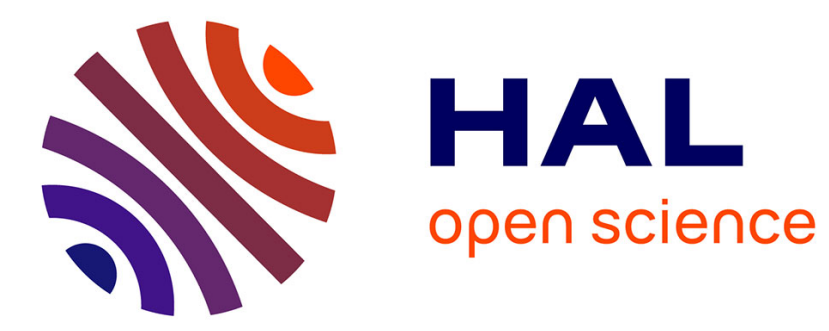

\title{
Analysis of a generalized kinematic impact law for multibody-multicontact systems, with application to the planar rocking block and chains of balls
}

Bernard Brogliato, Zhang Hongjian, Caishan Liu

\section{- To cite this version:}

Bernard Brogliato, Zhang Hongjian, Caishan Liu. Analysis of a generalized kinematic impact law for multibody-multicontact systems, with application to the planar rocking block and chains of balls. Multibody System Dynamics, 2012, 27 (3), pp.351-382. 10.1007/s11044-012-9301-3 . hal-00825573

\section{HAL Id: hal-00825573 \\ https://hal.inria.fr/hal-00825573}

Submitted on 3 Nov 2017

HAL is a multi-disciplinary open access archive for the deposit and dissemination of scientific research documents, whether they are published or not. The documents may come from teaching and research institutions in France or abroad, or from public or private research centers.
L'archive ouverte pluridisciplinaire HAL, est destinée au dépôt et à la diffusion de documents scientifiques de niveau recherche, publiés ou non, émanant des établissements d'enseignement et de recherche français ou étrangers, des laboratoires publics ou privés. 


\title{
Analysis of a generalized kinematic impact law for multibody-multicontact systems, with application to the planar rocking block and chains of balls
}

\author{
Bernard Brogliato $\cdot$ Hongjian Zhang • Caishan Liu
}

\begin{abstract}
In this paper, we analyze the capabilities of a generalized kinematic (Newton's like) restitution law for the modeling of a planar rigid block that impacts a rigid ground. This kinematic restitution law is based on a specific state transformation of the Lagrangian dynamics, using the kinetic metric on the configuration space. It allows one to easily derive a restitution rule for multiple impacts. The relationships with the classical angular velocity restitution coefficient $r$ for rocking motion are examined in detail. In particular, it is shown that $r$ has the interpretation of a tangential restitution coefficient. The case when Coulomb's friction is introduced at the contact impulse level together with an angular velocity restitution is analyzed. A simple chain of aligned balls is also examined, illustrating that the impact law applies to various types of multibody systems.
\end{abstract}

Keywords Multiple impacts · Rocking block - Kinetic angle $\cdot$ Kinematic restitution law · Housner model $\cdot$ Chains of balls $\cdot$ Moreau's impact law $\cdot$ Coulomb's friction

\section{Introduction}

Modeling the dynamics of a rigid block hitting a rigid ground has attracted the attention of scientists in the field of earthquake engineering for a long time [36, 46]; see, e.g., [2, 15, 27, $30,45,48,52,54,55]$ to cite a few recent works. This is also of interest for the study of

B. Brogliato $\cdot$ H. Zhang

BIPOP project-team, ZIRST Montbonnot, INRIA, 655 avenue de l'Europe, 38334 Saint Ismier, France e-mail: bernard.brogliato@inria.fr

H. Zhang

e-mail: zhanghj@pku.edu.cn

H. Zhang · C. Liu

State Key Laboratory for Turbulence and Complex Systems, College of Engineering, Peking University, Beijing 100871, People's Republic of China

C. Liu

e-mail: liucs@pku.edu.cn 
blocks falling on very steep planes in the mountain [26], and whose trajectories need to be estimated with sufficient accuracy. In parallel, the field of impact dynamics has witnessed an intense activity in the past 25 years; see, e.g. [5, 6, 8, 11-13, 16-20, 22-25, 38, 47, 49, 50] and references therein. It happens that the problem of modeling impacts with friction is a tough issue, especially when there are several simultaneous contact points and when friction is present during the impacts (multiple impacts with friction). Typically the so-called rocking block problem involves double-impacts with friction, when one assumes that the base contacts the ground at two points only. Together with chains of balls (Newton's cradles), the rocking block is an apparently simple multibody system (the block and the ground), however, it involves multiple impacts with friction and its modeling is consequently not simple at all. In this paper, we analyze the capabilities of a generalized kinematic restitution law, i.e., a restitution law based on restitution coefficients which are defined as ratios of post and preimpact velocities. The relationships with the classical angular velocity restitution law introduced in [27] and used since then in many papers on the rocking block are studied. We also examine a simple case of granular matter, which proves that the proposed generalized restitution law (of which the well-known Moreau's law is a particular case) can be applied to general multibody systems with simultaneous contacts/impacts.

The issue that is studied in this article may be seen as follows. Consider a rigid rectangular block made of homogeneous material, with its gravity center that coincides with its geometric center. Assume that the whole system is planar. Let the block collide a massive ground in a configuration such that the line passing through the gravity center and the contact point, is normal to the ground surface. This is a collinear impact where friction plays no role. This simple experiment may be used to estimate a coefficient of restitution between the block and the ground, at the block's corners. If both bodies are made of hard steel or of stone (granite) then the coefficient of restitution will be close to 1, typically around 0.9 . Moreover, it cannot be larger than 1, from a classical energetical constraint on frictionless impacts between two bodies [8]. Now consider the same block and suppose that the ratio height/width is large, i.e., it is a slender block. Typically, this ratio should be larger than 8 . Initialize the block with one corner in contact with the ground, the other corner being airborne, and let the block rotate around the contact corner until it hits the ground. If friction is large enough, both contact corners tangentially stick, and they also undergo plastic impacts with no rebound in the normal direction, while the block starts rocking until it stabilizes on the ground in a vertical position. Such behaviors are observed in experimental studies [30]. There is an apparent paradox that the simple impact at one corner, and the double impacts during the rocking motion, seem not to behave consistently one with respect to each other. Similar reasoning may be led for chains of balls. In this paper, we are not going to provide a complete answer to these paradoxes (which originate from the flexibilities which are present in the systems and are responsible for the energy dispersion and wave propagation), but we are rather going to study their consequences on kinematic impact laws.

The paper is organized as follows: In Sect. 2, the generalized impact law and the block's dynamics are presented. The detailed analysis of the kinematic laws for the planar block performing perfect rocking is made in Sect. 3. A summary is proposed in Sect. 4. An extension of the generalized kinematic law is studied in Sect. 5 which shows that the coefficients of restitution may not be uniquely defined from the energetical constraints. A simple case of granular system is presented in Sect. 7. Conclusions end the paper in Sect. 8.

Notations $A^{T}$ denotes the transpose of a matrix $A$. Let $f: \mathbb{R}^{n} \rightarrow \mathbb{R}^{m}$ be differentiable. The matrix $\nabla f(q)=\left({\frac{\partial f_{1}}{\partial q}}^{T}(q),{\frac{\partial f_{2}}{\partial q}}^{T}(q), \ldots,{\frac{\partial f_{m}}{\partial q}}^{T}(q)\right) \in \mathbb{R}^{n \times m}$ is its euclidean gradient, where 
$\frac{\partial f_{i}}{\partial q}(q)=\left(\frac{\partial f_{i}}{\partial q_{1}}(q), \frac{\partial f_{i}}{\partial q_{2}}(q), \ldots, \frac{\partial f_{i}}{\partial q_{n}}(q)\right) \in \mathbb{R}^{1 \times n}, i \in\{1, \ldots, m\}$. The $n \times n$ matrix $\operatorname{diag}\left(e_{i}\right)$ denotes a diagonal matrix with diagonal entries equal to $e_{i}, 1 \leq i \leq n . \lambda_{\max }(A)$ is the maximum eigenvalue of a matrix $A$. For two matrices $A$ and $B, A>B$ means that $A-B$ is positive definite (and so on for positive semidefinite, negative definite).

\section{The generalized kinematic restitution approach}

In this section, we consider the block as a Lagrangian system subject to unilateral constraints in its configuration space. The objective of this paper is the modeling of multiple impacts, which we precisely define now.

Definition 1 Let a mechanical Lagrangian system have generalized coordinates $q \in \mathbb{R}^{n}$, and a configuration space $\mathcal{C} \subseteq \mathbb{R}^{n}$. Let it be subject to a set of unilateral constraints $f_{i}(q) \geq 0,1 \leq i \leq m$, where the functions $f_{i}$ are continuously differentiable. We denote $\Sigma_{i}=\left\{q \in \mathbb{R}^{n} \mid f_{i}(q)=0\right\}$ the co-dimension one constraints boundaries. The admissible domain is therefore a finitely represented set $\Phi=\left\{q \in \mathbb{R}^{n} \mid f_{i}(q) \geq 0,1 \leq i \leq m\right\} \subseteq \mathcal{C}$. The boundary $\operatorname{Bd}(\Phi)$ of $\Phi$ is the union of facets $\bar{\Sigma}_{i} \subseteq \Sigma_{i}, 1 \leq i \leq m$, whose boundaries are made of codimension $p$ subspaces that correspond to intersections between $p$ surfaces $\Sigma_{i}$. A collision with such a codimension $p$ surface of $\operatorname{Bd}(\Phi)$ is a multiple impact of order $p$, or a $p$-impact.

When a $p$-impact occurs, the system undergoes $p$ simultaneous impacts at $p$ different points. The planar block will undergo simple impacts (1-impacts) and 2-impacts.

\subsection{The block dynamics}

Let us consider the block as a three degrees-of-freedom planar homogeneous solid, with generalized coordinates $q^{T}=(x, y, \theta)$, where $x$ and $y$ and the horizontal and vertical positions of the center of gravity $G, \theta$ is the angular position; see Fig. 1. Following [8, Chap. 6] we infer that the block, when $y \leq \sqrt{l^{2}+L^{2}}$, is subject to two unilateral constraints:

$$
\left\{\begin{array}{l}
f_{1}(q)=\theta+\theta_{\max }(y) \geq 0 \\
f_{2}(q)=-\theta+\theta_{\max }(y) \geq 0
\end{array}\right.
$$

Fig. 1 The block

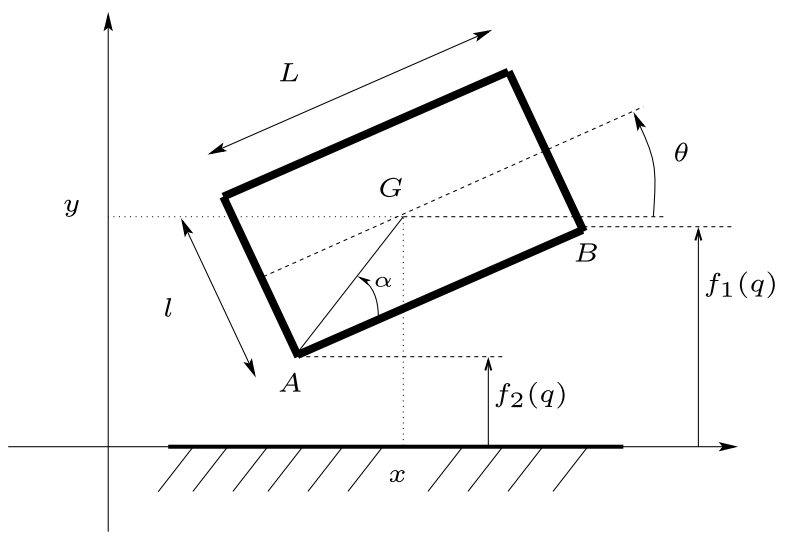


where $\theta_{\max }=-\arccos \left(\frac{2 y}{\sqrt{l^{2}+L^{2}}}\right)+\arctan \left(\frac{L}{l}\right)$. These two constraints can be equivalently written as

$$
\left\{\begin{array}{l}
f_{1}(q)=y-\frac{l}{2} \cos (\theta)+\frac{L}{2} \sin (\theta) \geq 0 \\
f_{2}(q)=y-\frac{l}{2} \cos (\theta)-\frac{L}{2} \sin (\theta) \geq 0
\end{array}\right.
$$

where $f_{1}(q) \geq 0$ expresses that point $B$ cannot penetrate into the ground, while $f_{2}(q) \geq 0$ expresses the same for point $A$. The underlying assumption is that the block/ground contact can be represented by the two points $A$ and $B$ at the corners. Obviously, when the block rotates by an angle larger than $\pm \frac{\pi}{2}$, the contact points change and one has to reconsider new unilateral constraints (this is what happens when so-called overturn occurs). The admissible domain defined by the unilateral constraints in the $(y, \theta)$ plane is not convex. A critical angle $\theta_{c}$ corresponds to a collinear single impact of the block at $A$ or $B$, with $A G$ (resp. $B G$ ) collinear to the normal to the ground, and therefore $\theta_{c}=\frac{\pi}{2}-\alpha$, where $\alpha$ is as depicted in Fig. 1. One expects that this is linked to the overturn of the block. At $\theta=0$ one has a 2-impact ( $A$ and $B$ hit the ground simultaneously). In a Lagrangian framework, this corresponds to the system hitting the admissible domain boundary at a codimension 2 surface. The dynamics of the block subject to frictionless constraints and gravity are:

$$
\left\{\begin{array}{l}
m \ddot{x}(t)=0 \\
m \ddot{y}(t)=\lambda_{n, 1}(t)+\lambda_{n, 2}(t)-m g \\
I_{G} \ddot{\theta}(t)=\lambda_{n, 1}(t)\left(\frac{l}{2} \sin (\theta(t))+\frac{L}{2} \cos (\theta(t))\right)+\lambda_{n, 2}(t)\left(\frac{l}{2} \sin (\theta(t))-\frac{L}{2} \cos (\theta(t))\right) \\
\quad 0 \leq \lambda_{n}(t) \perp f(q(t)) \geq 0
\end{array}\right.
$$

where the complementarity conditions are componentwise, with $f(q)^{T}=\left(f_{1}(q), f_{2}(q)\right)$ and $\lambda_{n}^{T}=\left(\lambda_{n, 1}, \lambda_{n, 2}\right)$. For a block with $G$ at the geometric center, one has $I_{G}=\frac{m}{12}\left(l^{2}+L^{2}\right)$. In (3), we have not yet considered the impacts with the ground, but only those phases of motion where the contact force is a bounded function of time. From (2) and (3), the Linear Complementarity Problem (LCP) that allows one to calculate the contact forces during the smooth phases of motion (i.e., outside impacts) is given by

$$
0 \leq \lambda_{n}(t) \perp \frac{d^{2}}{d t^{2}} f(q(t))=A(\theta) \lambda_{n}+B(\theta, \dot{\theta}) \geq 0
$$

with

$$
\begin{aligned}
& A(\theta)=\left(\begin{array}{cc}
\frac{1}{m}+\frac{1}{4 I_{G}}(l \sin (\theta)+L \cos (\theta))^{2} & \frac{1}{m}+\frac{1}{4 I_{G}}\left(l^{2} \sin ^{2}(\theta)-L^{2} \cos ^{2}(\theta)\right) \\
\frac{1}{m}+\frac{1}{4 I_{G}}\left(l^{2} \sin ^{2}(\theta)-L^{2} \cos ^{2}(\theta)\right) & \frac{1}{m}+\frac{1}{4 I_{G}}(l \sin (\theta)-L \cos (\theta))^{2}
\end{array}\right), \\
& A(\theta)=A^{T}(\theta), \quad B(\theta, \dot{\theta})=\left(\begin{array}{cc}
-g+\frac{1}{2} \dot{\theta}^{2}(l \cos (\theta)-L \sin (\theta)) \\
-g+\frac{1}{2} \dot{\theta}^{2}(l \cos (\theta)+L \sin (\theta))
\end{array}\right) .
\end{aligned}
$$

One calculates that $\operatorname{det}(A(\theta))=\frac{L^{2}}{m I_{G}} \cos ^{2}(\theta)$ so that $A(\theta)$ is symmetric positive definite except at $\theta= \pm \frac{\pi}{2}$. These values are, however, outside the range of block orientations within which the analysis is done. We conclude that for all angles $\theta \in\left(-\frac{\pi}{2}, \frac{\pi}{2}\right) A(\theta)>0$ and the normal contact force $\lambda_{n}$ can be computed uniquely as the solution of the LCP in (4) whatever $\theta$ and $\dot{\theta}$; see for instance [1, Theorem B.2]. $A(\theta)$ is the so-called Delassus' matrix of the 
system (2), (3). When tangential effects act at the contact points the dynamics in (3) becomes

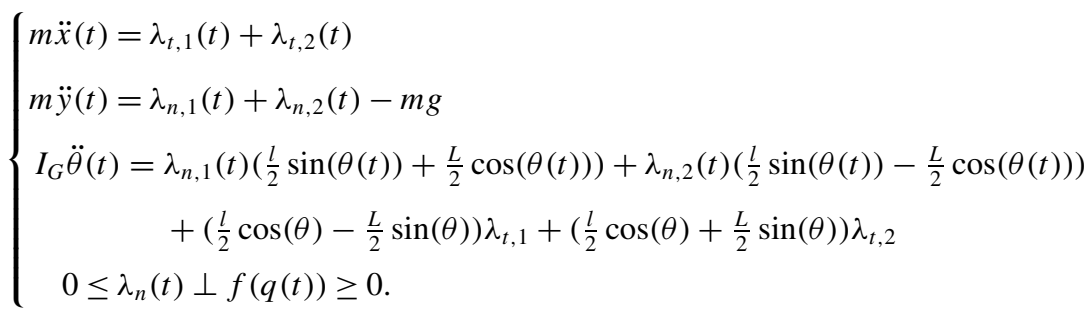

One can rewrite (5) more compactly as follows:

$$
M \ddot{q}(t)=W_{n}(q(t)) \lambda_{n}(t)+W_{t}(q(t)) \lambda_{t}(t)-\mathbf{g}
$$

where the various terms can be easily identified. In particular $W_{n}(q)=\nabla f(q)$. If the contact point $i$ detaches then the complementarity conditions imply that $\lambda_{n, i}=0$ and the tangential model is supposed to guarantee $\lambda_{t, i}=0$. The tangential effects may be due to Coulomb friction, in which case $\lambda_{t, i}(t) \in-\mu_{i} \lambda_{n, i}(t) \operatorname{sgn}\left(v_{t, i}(t)\right), i=1,2$, where $\mu_{i}>0$ is the friction coefficient at contact $i$, and $v_{t, i}$ is the tangential (more generally, horizontal) velocity at the point $i$, i.e., $v_{t, 1}=\dot{x}+\left(\frac{l}{2} \cos (\theta)-\frac{L}{2} \sin (\theta)\right) \dot{\theta}$ at $B$ and $v_{t, 2}=\dot{x}+\left(\frac{l}{2} \cos (\theta)+\frac{L}{2} \sin (\theta)\right) \dot{\theta}$ at $A$ (from which $v_{t, 1}=v_{t, 2}$ when $\theta=0$ ). The dynamics in (5) stands for fixed ground. If the base has a horizontal motion denoted as $x_{b}(t)$, then the friction law is changed to $\lambda_{t, i}(t) \in-\mu_{i} \lambda_{n, i}(t) \operatorname{sgn}\left(v_{t, i}(t)-v_{b}(t)\right), i=1,2$, where $v_{b}(t)$ is the base horizontal velocity.

\subsection{A generalized kinematic restitution mapping}

We take it as granted here that the positions are continuous functions of time while the velocities are right-continuous of local bounded variations, with possible discontinuities at the impact times, see [3, 14, 39] for more details on such system's well-posedness. Let us introduce a specific change of generalized velocities [8, Chap. 6], [9] (see also [37] for similar tools) for an $n$-dimensional Lagrangian system subject to $m$ unilateral constraints $f_{i}(q) \geq 0,1 \leq i \leq m$, that we suppose to be mutually independent, and with a symmetric positive definite mass matrix $M(q)$. The vector $\mathbf{n}_{q, i}=\frac{M^{-1}(q) \nabla f_{i}(q)}{\sqrt{\nabla f_{i}(q)^{T} M^{-1}(q) \nabla f_{i}(q)}}$ denotes the unitary normal vector to the surface $\Sigma_{i}=\left\{q \in \mathbb{R}^{n} \mid f_{i}(q)=0\right\}$, in the kinetic metric. ${ }^{1}$ One may form a basis by completing the $\mathbf{n}_{q, i}$ normal vectors with $n-m$ mutually independent tangential vectors $\mathbf{t}_{q, i}$ such that $\mathbf{n}_{q, i}^{T} M(q) \mathbf{t}_{q, j}=0$ for all $i \in\{1, \ldots, m\}$ and $j \in\{m+1, \ldots, n\}$, and such that $\mathbf{t}_{q, j}^{T} M(q) \mathbf{t}_{q, j}=1$. We have thus constructed a basis in the configuration space $\mathcal{C}$ of the system, at the point $q$ on the boundary of the admissible domain $\Phi=\left\{q \in \mathcal{C} \subseteq \mathbb{R}^{n} \mid f_{i}(q) \geq 0,1 \leq i \leq m\right\}$. More precisely, the vectors $\mathbf{n}_{q, i}$ generate the normal cone to $\Phi$ at $q$ in the kinetic metric, which is $M^{-1}(q) N_{\Phi}(q)$ (see e.g. [1, Appendix A]), whereas the vectors $\mathbf{t}_{q, i}$ form a basis of the tangent space $T_{q} \mathcal{C}$ to $\mathcal{C}$ at $q$. Then $\Xi(q)=\left(\begin{array}{c}\mathbf{n}_{q}^{T} \\ \mathbf{t}_{q}^{T}\end{array}\right)$ is an $n \times n$ full-rank matrix,

$$
\mathbf{n}_{q}^{T}=\left(\begin{array}{c}
\mathbf{n}_{q, 1}^{T} \\
\vdots \\
\mathbf{n}_{q, m}^{T}
\end{array}\right), \quad \mathbf{t}_{q}^{T}=\left(\begin{array}{c}
\mathbf{t}_{q, m+1}^{T} \\
\vdots \\
\mathbf{t}_{q, n}^{T}
\end{array}\right)
$$

\footnotetext{
${ }^{1}$ The kinetic metric is the metric defined with the mass matrix $M(q)=M^{T}(q)>0$, such that for two vectors $x$ and $y$ the inner product is $x^{T} M(q) y$.
} 
and we may define the full-rank $n \times n$ transformation matrix $\mathcal{M}(q)=\Xi(q) M(q)$. The transformed velocities are given by

$$
\left(\begin{array}{c}
\dot{q}_{\text {norm }} \\
\dot{q}_{\text {tan }}
\end{array}\right)=\mathcal{M}(q) \dot{q}
$$

where a normal and a tangential component appears. Notice that $\dot{q}_{\text {norm }, i}=$ $\frac{\nabla f_{i}^{T}(q) \dot{q}}{\sqrt{\nabla f_{i}(q)^{T} M^{-1}(q) \nabla f_{i}(q)}}$ The Lagrangian dynamics is then transformed into [8, Sect. 6.2] [9]:

$$
\left(\begin{array}{c}
\ddot{q}_{\mathrm{norm}} \\
\ddot{q}_{\mathrm{tan}}
\end{array}\right)+F(q, \dot{q})=\left(\begin{array}{c}
\mathbf{n}_{q}^{T} \nabla f(q) \lambda_{n} \\
0
\end{array}\right)
$$

where it is still assumed that the constraints are frictionless in (8). In case some tangential forces act on the system then from (6). one finds that the right-hand side of (8) is equal to $\left(\begin{array}{c}\mathbf{n}_{q}^{T} \nabla f(q) \lambda_{n}+\mathbf{n}_{q}^{T} W_{t}(q) \lambda_{t} \\ \mathbf{t}_{q}^{T} W_{t}(q) \lambda_{t}\end{array}\right)$, since $\mathbf{t}_{q}^{T} W_{n}(q)=\mathbf{t}_{q}^{T} M(q) \mathbf{n}_{q}=0$, however, in general $\mathbf{n}_{q}^{T} W_{t}(q)$ is nonzero (this will be the case for the rocking block; this is also the case of a slender rod sliding on a rough ground; see, e.g., [8, Sect. 5.5]). In other words, the normal force does not act in the tangential direction, but tangential forces may act in the normal direction. This is the source of difficulties like the Painlevé paradoxes in contact mechanics [8]. The term $F(q, \dot{q})$ gathers smooth, bounded functions only. The advantage of this velocity transformation is that the system in (8) appears to be decoupled because the normal contact force does not act in the tangential direction. The dynamics in (8) is not in a Lagrangian formalism, however, because in general the above velocity transformation does not correspond to a generalized coordinate transformation derivative. Notice that $\mathbf{n}_{q}^{T} \nabla f(q)$ is the Delassus' matrix with normalizations of the components. If all the constraints are pairwise orthogonal in the kinetic metric, then $\mathbf{n}_{q}^{T} \nabla f(q)=\operatorname{diag}\left(\sqrt{\nabla f_{i}^{T}(q) M^{-1}(q) \nabla f_{i}(q)}\right)$ : the normal directions are also decoupled. In such a case, the kinetic angles between all the couples of surfaces, i.e., $\theta_{i j}=\left(\widehat{\Sigma_{i}, \Sigma_{j}}\right)_{M(q)}, i \neq j$, are equal to $\frac{\pi}{2}$. We recall that $\theta_{i j}=\pi-\arccos \frac{\mathbf{n}_{q, i}^{T} M(q) \mathbf{n}_{q, j}}{\left\|\mathbf{n}_{q, i} \mid\right\|_{M(q)}\left\|\mathbf{n}_{q, j}\right\|_{M(q)}}$ so: ${ }^{2}$

$$
\theta_{i j}=\pi-\arccos \frac{\nabla f_{i}(q)^{T} M^{-1}(q) \nabla f_{j}(q)}{\sqrt{\nabla f_{i}(q)^{T} M^{-1}(q) \nabla f_{i}(q)} \sqrt{\nabla f_{j}(q)^{T} M^{-1}(q) \nabla f_{j}(q)}} .
$$

Kinetic angles are quantities that reflect the couplings between the inertial properties and the geometrical properties of the system with unilateral constraints. They are known to play a significant role in the dynamics of such systems, with a strong influence on the continuity of trajectories with respect to initial data [1, 3, 21, 41]. For the block/ground system, the kinetic angle $\theta_{12}$ between the two constraints is given by

$$
\theta_{12}=\pi-\arccos \left(\frac{l^{2}-2 L^{2}}{l^{2}+4 L^{2}}\right)
$$

at $\theta=0$. Denoting the aspect ratio $a=\frac{l}{L}$ we may rewrite it as $\theta_{12}=\pi-\arccos \left(\frac{a^{2}-2}{a^{2}+4}\right)$ : there is a one-to-one correspondence between $a$ and $\theta_{12}$. It satisfies $\theta_{12}=\frac{\pi}{2}$ if $l=\sqrt{2} L$,

\footnotetext{
${ }^{2}$ The kinetic angle is obtained after substraction from $\pi$ because the normal vectors point outside the admissible domain of the configuration space.
} 
$0<\theta_{12}<\frac{\pi}{2}$ if $0<l<\sqrt{2} L$ (flat block), and $\pi>\theta_{12}>\frac{\pi}{2}$ if $l>\sqrt{2} L$ (slender block). When $a$ varies from 0 (infinitely flat block with infinite width $L$ ) to $+\infty$ (infinitely slender block with infinite height $l$ ) then $\theta_{12}$ varies from $\frac{\pi}{3}$ to $\pi$. One expects that $\theta_{12} \in\left[\frac{\pi}{3}, \pi\right]$ implies a rich dynamical behavior for the block, depending on its aspect ratio.

Remark 1 The calculations are made in this paper for a perfect rectangular homogeneous block. It is clear that all the calculations can easily be done for more general blocks when for instance the center of mass and the geometric center do not coincide, or when the block's geometry is not rectangular. Since most of the literature on the topic deals with perfect blocks, we, however, lead our developments with such an assumption, keeping in mind that generalizations are quite possible. For instance all the calculations that are made next in this paper could be led keeping $I_{G}$ and not specifying its value. The interest of the kinetic angle, compared to the aspect ratio, is that this is a general notion which could be applied to study more general blocks. Moreover, it is the quantity that is used in theoretical studies involving several unilateral constraints $[3,41]$. Finally, it is clear from (8) that kinetic angles naturally appear in impact dynamics.

At an impact time, we deduce from (8), the contact force being a Dirac measure $\lambda_{n}=$ $p_{n}(t) \delta_{t}$, that:

$$
\left\{\begin{array}{l}
\dot{q}_{\text {norm }}\left(t^{+}\right)-\dot{q}_{\text {norm }}\left(t^{-}\right)=\mathbf{n}_{q}^{T} \nabla f(q) p_{n}(t) \\
\dot{q}_{\tan }\left(t^{+}\right)-\dot{q}_{\tan }\left(t^{-}\right)=0
\end{array}\right.
$$

which is similar to the impact dynamics of a particle hitting a frictionless wall, where the normal and the tangential directions are decoupled. The percussion $p_{n}(t)$ is the density of the measure $\lambda_{n}$ at time $t$, and is indeed a function of time. Then it is natural to mimic the case of a particle and to define a generalized Newton's (or kinematic) restitution rule as

$$
\dot{q}_{\text {norm }}\left(t^{+}\right)=-\mathcal{E}_{n} \dot{q}_{\text {norm }}\left(t^{-}\right) \quad \dot{q}_{\text {norm }, \mathrm{i}}\left(t^{-}\right) \leq 0 \quad i \in\{1, \ldots, m\}
$$

at an impact time $t$ where all the $m$ surfaces are collided at the same time, where $t^{+}$and $t^{-}$indicate the right and left limits as usual. The matrix $\mathcal{E}_{n} \in \mathbb{R}^{m \times m}$ is a matrix of normal restitution. From (11), it follows that $\dot{q}_{\tan }$ is continuous at such $t$. It is easy to compute that the loss of kinetic energy is given by

$$
T_{L}(t)=T\left(t^{+}\right)-T\left(t^{-}\right)=\frac{1}{2} \dot{q}_{\text {norm }}^{T}\left(t^{-}\right)\left(\mathcal{E}_{n}^{T} A(q) \mathcal{E}_{n}-A(q)\right) \dot{q}_{\text {norm }}\left(t^{-}\right)
$$

where $A(q)=\mathbf{n}_{q}^{T} M(q) \mathbf{n}_{q} \in \mathbb{R}^{m \times m}$ is symmetric positive definite since the constraints are supposed to be functionally independent. Obviously, if $m=1$ then $A(q)=1, \mathcal{E}_{n}=e_{n}$ and (13) boils down to the classical energy loss for two frictionless bodies colliding at one point [8, Eqs. (4.44) and (6.13)]. From the energetic constraint $T_{L}(t) \leq 0$, one finds that $\mathcal{E}_{n}^{T} A(q) \mathcal{E}_{n} \leq A(q)$ is sufficient to guarantee the kinetic energy loss. We have the following proposition.

Proposition 1 Suppose that $\mathcal{E}_{n}=\mathcal{E}_{n}^{T}$. Then a sufficient condition for $T_{L}(t) \leq 0$ in (13) for any vector $\dot{q}_{\text {norm }}\left(t^{-}\right)$is that $\left|\lambda_{\max }\left(\mathcal{E}_{n}\right)\right| \leq 1$.

Proof $T_{L}(t) \leq 0$ holds for any $\dot{q}_{\text {norm }}\left(t^{-}\right)$provided $\mathcal{E}_{n}^{T} A(q) \mathcal{E}_{n} \leq A(q)$, that is equivalent to $\lambda_{\max }\left(A^{-\frac{1}{2}}(q) \mathcal{E}_{n}^{T} A(q) \mathcal{E}_{n} A^{-\frac{1}{2}}(q)\right) \leq 1$, using Proposition 8.1.2 xi) and xiv) and Lemma 8.4.1 
iii) in [4]. Let us denote $B(q)=A^{-\frac{1}{2}}(q) \mathcal{E}_{n} A^{-\frac{1}{2}}(q)$. The matrix $B(q)$ is similar to the matrix $\mathcal{E}_{n}$, so they have the same eigenvalues (see [28, Proposition 1, p. 152]). Therefore, the condition $\lambda_{\max }\left(B^{2}(q)\right) \leq 1$ is equivalent to $\lambda_{\max }\left(\mathcal{E}_{n}^{2}\right) \leq 1$. The result follows.

These conditions are sufficient for the energetical consistency of the impact law, independently of the preimpact velocity $\dot{q}_{\text {norm }}\left(t^{-}\right)$. It is important that they hold independently of the inertia parameters, hence extending the case $m=1$. This independence also holds in cases where $A(q)$ itself does not depend on the entries of $M(q)$, as is the case for instance for the monodisperse chain of balls example in Sect. 7. The nonpositiveness of $\mathcal{E}_{n}^{T} A(q) \mathcal{E}_{n}-A(q)$ can be relaxed to the copositivity of $\mathcal{E}_{n}^{T} A(q) \mathcal{E}_{n}-A(q)$ on $\mathbb{R}_{-}^{m}$, because each component of $\dot{q}_{\text {norm }}\left(t^{-}\right)$is nonpositive. However, for particular preimpact velocities the conditions may change; see Sects. 3, 5, and 7. Since an impact has occurred with all $m$ surfaces, one has $\dot{q}_{\text {norm }, i}\left(t^{-}\right)<0$ for all $1 \leq i \leq m$. The post-impact velocity has to be admissible, i.e., it has to point inside the admissible domain $\Phi$ : This is a kinematic constraint. In other words $\dot{q}_{\text {norm }, i}\left(t^{+}\right) \geq 0$ for all $1 \leq i \leq m$. If $\mathcal{E}_{n}$ is diagonal with $m$ nonnegative coefficients $e_{n, i}$, then this implies that $e_{n, i} \geq 0$, whereas the energetic constraint implies that $-1 \leq e_{n, i} \leq 1$. We deduce that the energetic and the post-impact velocity admissibility imply that $e_{n, i} \in[0,1]$ when $\mathcal{E}_{n}=\operatorname{diag}\left(e_{n, i}\right)$. A third constraint is the form of the contact force (and of its impulse as a consequence), that we name the kinetic constraint. In (8), we have supposed that the constraints are frictionless, i.e., there are no contact forces in the generalized tangential direction. This means that the right-hand side is as in (11), and it implies that $\dot{q}_{\tan }$ is continuous at the impact time. One also has an additional kinetic constraint of positivity $p_{n}(t) \geq 0$ (componentwise).

Remark 2 Remind that there is no sign condition to be imposed neither to $\lambda_{t, 1}$ and $\lambda_{t, 2}$, nor to $p_{t, 1}$ and $p_{t, 2}$.

It is obvious that by modifying the kinetic constraint (i.e., the form of the contact force impulse) one may also define a general restitution matrix $\mathcal{E}=\left(\begin{array}{cc}\mathcal{E}_{n} & 0 \\ 0 & \mathcal{E}_{\text {tan }}\end{array}\right) \in \mathbb{R}^{n \times n}$ such that

$$
\left(\begin{array}{c}
\dot{q}_{\text {norm }}\left(t^{+}\right) \\
\dot{q}_{\text {tan }}\left(t^{+}\right)
\end{array}\right)=-\mathcal{E}\left(\begin{array}{c}
\dot{q}_{\text {norm }}\left(t^{-}\right) \\
\dot{q}_{\text {tan }}\left(t^{-}\right)
\end{array}\right)
$$

and $\dot{q}_{\text {tan }}$ may jump, with generalized tangential restitution coefficients $e_{t, i}, m+1 \leq i \leq n$. In such a case, one has to take into account the tangential impulse $p_{t}(t)$ and modify the right-hand side of (11) accordingly as $\left(\begin{array}{c}\mathbf{n}_{q}^{T} \nabla f(q) p_{n}(t)+\mathbf{n}_{q}^{T} W_{t}(q) p_{t}(t) \\ \mathbf{t}_{q}^{T} W_{t}(q) p_{t}(t)\end{array}\right)$. By suitably varying the coefficients of $\mathcal{E}$, then the whole space of admissible post-impact velocities can be spanned, which is considered as a nice property for a restitution law [8, 10, 22, 23]. An example of such a restitution matrix will be given in Remark 7. In the case with no tangential effects, one has $\mathcal{E}=\left(\begin{array}{cc}\mathcal{E}_{n} & 0 \\ 0 & -I_{n-m}\end{array}\right)$.

Remark 3 The generalized impact law in (14) has been introduced in [9, Chap. 6], where the three consistency constraints (kinematic, kinetic, energetic) are clearly stated (see also [23, Sect. 5.2]). The above velocity decomposition and restitution rule is interesting because it allows one to decouple the normal and tangential directions for a Lagrangian system in its configuration space. Moreover, it may be given several nice interpretations following the developments in [22, 23]. First notice that $\dot{q}=\sum_{i=1}^{m} \dot{q}_{\mathrm{norm}, i} \mathbf{n}_{q, i}+\sum_{i=m+1}^{n} \dot{q}_{\mathrm{tan}, i} \mathbf{t}_{q, i}$. This decomposition is the same as the one in [22, Sect. 6] and it follows in particular that the impact 
law in (12) is equivalent to Moreau's impact law with a global dissipation index $e \in[0,1]$, provided the kinetic constraint is properly chosen and $\mathcal{E}_{n}=\operatorname{diag}(e)$ (see, for instance, Eqs. (5.15) and (5.16) in [23], where the set denoted $\mathcal{T}_{\mathcal{C}}^{\perp}(q)$, and which is equal to $M^{-1}(q) N_{\Phi}(q)$, is generated by the vectors $\left.\mathbf{n}_{q, i}\right)$. Moreau's kinematic impact law is known not to cover the whole set of admissible post-impact velocities (see, e.g., Fig. 11 in [22], see also [23]). For this reason, Frémond $[19,20]$ advocated that some "distance effects" should be incorporated into the collision rule and defined off-diagonal coefficients, yielding what was named after the Frémond matrices [23, 24, 42]. Actually (as far as chains of balls are concerned), the Frémond matrix and $\mathcal{E}_{n}$ are similar. It is important, however, to keep in mind that these developments hold as long as the constraints are perfect, i.e., there are no tangential forces at the contact points. One objective of this paper is to study what happens when tangential effects are added at the impacts.

\section{Application to the block impact dynamics}

Let us see now how the above material may be applied to the particular case of the planar block. More specifically, in this section, we will investigate how the rocking motion of the block may be modeled with the above generalized kinematic law. The rocking motion is an interesting one, because it involves a double-impact each time one of the corners hits the ground. In this paper, we will focus on several particular motions of the block: free rocking with sliding contact points, half-rocking, free-rocking with sticking contact points. Each one of these motions implies a set of kinematic constraints (equality constraints on the velocities). From (5), it follows that the impact dynamics of the block is given by

$$
\left\{\begin{array}{l}
m\left(\dot{x}\left(t^{+}\right)-\dot{x}\left(t^{-}\right)\right)=p_{t, 1}(t)+p_{t, 2}(t) \\
m\left(\dot{y}\left(t^{+}\right)-\dot{y}\left(t^{-}\right)\right)=p_{n, 1}(t)+p_{n, 2}(t) \\
I_{G}\left(\dot{\theta}\left(t^{+}\right)-\dot{\theta}\left(t^{-}\right)\right)=\frac{L}{2}\left(p_{n, 1}(t)-p_{n, 2}(t)\right)+\frac{l}{2}\left(p_{t, 1}(t)+p_{t, 2}(t)\right)
\end{array}\right.
$$

where $t$ is the impact time, $\theta(t)=0$ at an impact time, and we recall that being the density of the measure $\lambda_{n}$ at time $t$, the impulse $p_{n}$ is a function of time. In the frictionless case (no tangential contact forces), one has $p_{t, 1}(t)+p_{t, 2}(t)=0$. Similarly to (5), it is assumed that $p_{n, i}=0 \Rightarrow p_{t, i}=0$. One can apply the restitution rule in (12) with $\mathcal{E}_{n}=\operatorname{diag}\left(e_{n, 1}, e_{n, 2}\right)$, so that there are five unknowns (three post-impact velocities and two impulses) and five equations. One has

$$
\begin{aligned}
\dot{q}_{\text {norm }, 1} & =\frac{\dot{y}+\left(\frac{l}{2} \sin (\theta)+\frac{L}{2} \cos (\theta)\right) \dot{\theta}}{\sqrt{\frac{1}{m}+\frac{1}{4 I_{G}}(l \sin (\theta)+L \cos (\theta))^{2}}} \\
\dot{q}_{\mathrm{norm}, 2} & =\frac{\dot{y}+\left(\frac{l}{2} \sin (\theta)-\frac{L}{2} \cos (\theta)\right) \dot{\theta}}{\sqrt{\frac{1}{m}+\frac{1}{4 I_{G}}(l \sin (\theta)+L \cos (\theta))^{2}}} \\
\dot{q}_{\mathrm{tan}} & =\sqrt{m} \dot{x} .
\end{aligned}
$$

A particularity of the block is, however, that except if (i) $\theta(t)=0$ in which case the two points $A$ and $B$ may hit the ground at the same time, there are only two possible other impact configurations: (ii) $A$ (resp. $B$ ) is in lasting contact and the block hits the ground at $B$ (resp. at $A$ ), (iii) $A$ (resp. $B$ ) hits the ground while $B$ (resp. $A$ ) is airborne. Let us study first the 
subcase of case (ii) that corresponds to a rocking motion of the block, i.e., when $B$ sticks after the shock while $A$ detaches from the ground. We shall call free-rocking the rocking motion when the base is fixed and the block evolves freely from some initial state. The subcase of case (ii) where $A$ (resp. $B$ ) keeps the contact after the shock and $B$ (resp. $A$ ) rebounds will be called the half-rocking motion. Notice that the fact that a point keeps the contact does not mean that it is fixed: it may slide on the constraint surface. This is why we will introduce sticking rocking and sliding rocking. Sticking rocking occurs when the contact points are tangentially fixed (a tangential kinematic constraint is imposed), while sliding rocking occurs for frictionless systems. Notice that when there is no tangential effects at the contact points, then $\dot{x}$ is constant so that the contact points necessarily slip on the ground when the block moves: free-rocking without friction occurs with slipping contact points. There are intermediate cases where the contact points may undergo stick and slip modes during impact and contact phases. However, in this paper, we shall focus only on the above two limited cases (sticking and sliding). The more general situation is examined in another paper, using another multiple impact model with friction introduced in [31-34, 58]. In the sequel, the capabilities of the generalized impact law are examined in detail, when various assumptions are made. This is a similar study as what has been previously done for the case of singleimpact laws, where the subspace of post-impact velocities may be studied [10]. In the case of multiple impacts without friction, a general framework was proposed in [22]. The classical Housner angular restitution coefficient will also be recast into our framework. Studying the relationships between various coefficients in kinematic approaches has been done for single impacts [6], and our work may be seen as an extension in a multiple impact framework.

\subsection{Diagonal $\mathcal{E}_{n}, p_{n, 1}(t) \neq 0, p_{n, 2}(t)=0$ (sliding rocking motion)}

Suppose that the block rotates around $A$ (i.e., $f_{2}(q)=0$ on a nonzero time interval, and we assume that $\lambda_{n, 2}$ is a bounded function of time, i.e., $\left.p_{n, 2}(t)=0\right)$ and that $B$ hits the ground and then sticks on it in the normal direction (it may slide). The fact that the contact force is not a Dirac measure at point $A$ at the impact time may appear as a natural assumption since the point $A$ is at rest on the ground. However, we are going to see that this is in fact a strong and stringent assumption for such a 2-impact, that imposes hard limitations on the post-impact velocities the impact law may span. At the impact time, one has $\theta(t)=0$ and $\dot{q}_{\text {norm }, 2}\left(t^{-}\right)=0$. One deduces that $\dot{y}\left(t^{-}\right)=\frac{L}{2} \dot{\theta}\left(t^{-}\right)$. The impact dynamics (15) together with (12) becomes

$$
\left\{\begin{array}{l}
m \dot{x}\left(t^{+}\right)=m \dot{x}\left(t^{-}\right) \\
m\left(\dot{y}\left(t^{+}\right)-\dot{y}\left(t^{-}\right)\right)=p_{n, 1}(t) \\
I_{G}\left(\dot{\theta}\left(t^{+}\right)-\dot{\theta}\left(t^{-}\right)\right)=\frac{L}{2} p_{n, 1}(t) \\
\dot{q}_{\text {norm }, 1}\left(t^{+}\right)=-e_{n, 1} \dot{q}_{\text {norm }, 1}\left(t^{-}\right) \\
\dot{q}_{\text {norm }, 2}\left(t^{+}\right)=-e_{n, 2} \dot{q}_{\text {norm }, 2}\left(t^{-}\right)=0
\end{array}\right.
$$

since $\dot{q}_{\text {norm }, 2}\left(t^{-}\right)=0$ and $\mathcal{E}_{n}$ is diagonal. Or equivalently (recall that $\theta(t)=0$ at the doubleimpact):

$$
\left\{\begin{array}{l}
\dot{q}_{\text {norm }}\left(t^{+}\right)-\dot{q}_{\text {norm }}\left(t^{-}\right)=\left(\begin{array}{ll}
\frac{4 L^{2}+l^{2}}{m\left(L^{2}+l^{2}\right)} & \frac{l^{2}-2 L^{2}}{m\left(l^{2}+L^{2}\right)} \\
\frac{l^{2}-2 L^{2}}{m\left(l^{2}+L^{2}\right)} & \frac{4 L^{2}+l^{2}}{m\left(L^{2}+l^{2}\right.}
\end{array}\right)\left(\begin{array}{l}
p_{n, 1}(t) \\
p_{n, 2}(t)
\end{array}\right) \\
\dot{q}_{\tan }\left(t^{+}\right)=\dot{q}_{\tan }\left(t^{-}\right) \\
\dot{q}_{\text {norm }, 1}\left(t^{+}\right)=-e_{n, 1} \dot{q}_{\text {norm }, 1}\left(t^{-}\right) \\
\dot{q}_{\text {norm }, 2}\left(t^{+}\right)=-e_{n, 2} \dot{q}_{\text {norm }, 2}\left(t^{-}\right)=0 .
\end{array}\right.
$$


From this, we infer that

$$
\dot{y}\left(t^{+}\right)=\frac{6 L^{2} e_{n, 1}+2 L^{2}-l^{2}}{4 L^{2}+l^{2}} \dot{y}\left(t^{-}\right)
$$

and

$$
\dot{\theta}\left(t^{+}\right)=-\frac{6 L^{2} e_{n, 1}+2 L^{2}-l^{2}}{4 L^{2}+l^{2}} \dot{\theta}\left(t^{-}\right) .
$$

Notice that the rocking motion implies that $e_{n, 1}=0$ because after the shock one has $f_{1}(q)=0$ and $\dot{q}_{\text {norm, } 1}\left(t^{+}\right)=0$ (the contact at $B$ is lasting), so that $\dot{y}\left(t^{+}\right)+\frac{L}{2} \dot{\theta}\left(t^{+}\right)=0$. This together with $\dot{q}_{\text {norm, } 2}\left(t^{+}\right)=0$ implies that $\dot{y}\left(t^{+}\right)=0$ and $\dot{\theta}\left(t^{+}\right)=0$, so that from (22) one has necessarily $l^{2}=2 L^{2}$. Also from (19), $p_{n, 1}(t)=-\frac{2 I_{G}}{L} \dot{\theta}\left(t^{-}\right)>0$ since $\dot{\theta}\left(t^{-}\right)<0$. We conclude that the impact map (12) with the assumption that $\lambda_{n, 2}$ is a bounded function of time cannot model the rocking motion of the block and models only the most dissipative motion when the block comes to rest after the impact, for the case that the kinetic angle is equal to $\frac{\pi}{2}$. From (22) and $e_{n, 1}=0$, one deduces that

$$
\dot{\theta}\left(t^{+}\right)=-\frac{2 L^{2}-l^{2}}{4 L^{2}+l^{2}} \dot{\theta}\left(t^{-}\right)
$$

and necessarily $l=\sqrt{2} L$. By symmetry of the system, we can do the same calculations when the block rotates around $B$ and hits the ground at $A$.

3.2 Diagonal $\mathcal{E}_{n}, p_{n, 1}(t) \neq 0, p_{n, 2}(t) \neq 0$ (sliding rocking block)

Let us now relax the assumption that $p_{n, 2}(t)=0$. The impact dynamics is

$$
\left\{\begin{array}{l}
m \dot{x}\left(t^{+}\right)=m \dot{x}\left(t^{-}\right) \\
m\left(\dot{y}\left(t^{+}\right)-\dot{y}\left(t^{-}\right)\right)=p_{n, 1}(t)+p_{n, 2}(t) \\
I_{G}\left(\dot{\theta}\left(t^{+}\right)-\dot{\theta}\left(t^{-}\right)\right)=\frac{L}{2}\left(p_{n, 1}(t)-p_{n, 2}(t)\right) \\
\dot{q}_{\mathrm{norm}, 1}\left(t^{+}\right)=-e_{n, 1} \dot{q}_{\mathrm{norm}, 1}\left(t^{-}\right) \\
\dot{q}_{\text {norm }, 2}\left(t^{+}\right)=0 .
\end{array}\right.
$$

Doing similar calculations as above, one deduces that $\dot{\theta}\left(t^{+}\right)=0$ and $\dot{y}\left(t^{+}\right)=0$ because for rocking to occur $e_{n, 1}=0$. One finds $p_{n, 1}(t)=-\left(\frac{m L}{4}+\frac{I_{G}}{L}\right) \dot{\theta}\left(t^{-}\right) \geq 0$, and $p_{n, 2}(t)=$ $m\left(\frac{l^{2}-2 L^{2}}{12 L}\right) \dot{\theta}\left(t^{-}\right)$. Thus, $p_{n, 2}(t) \geq 0$ if and only if $l^{2} \leq 2 L^{2}$ (the kinetic angle at $\theta=0$ is less than or equal to $\frac{\pi}{2}$ ). This means that according to this model, both percussions are nonzero while the block is in a motion like (ii) above, only if the block is of the flat type.

Remark 4 As pointed out in Remark 3, the case analyzed in Sects. 3.1 and 3.2 are equivalent to Moreau's impact rule. A similar analysis is made in [23, Sect. 5.8], where the rocking rod is studied (the rock and the rod being different systems yield different behaviors, however). It is known that Moreau's impact rule has to be modified with supplementary coefficients in order to allow for rocking [40, Sect. 6]. Limitations of this impact rule for chains of balls are also studied in $[23$, Sects. 3.8, 5.6]; see Sect. 7. 


\subsection{General $\mathcal{E}_{n}, p_{n, 1}(t) \neq 0, p_{n, 2}(t) \neq 0$ (sliding rocking block)}

One concludes that the kinematic model of restitution with a diagonal $\mathcal{E}_{n}$ is a quite poor model that allows one to cover only very few cases of rock motion. In order to enlarge the set of reachable post-impact velocities, let us investigate a more general impact law. In particular, we may set $\mathcal{E}_{n}$ with off-diagonal elements, i.e., we introduce couplings between the two normal directions at the impact times. Let us set $\mathcal{E}_{n}=\left(\begin{array}{cc}e_{n, 1} & e_{n, 12} \\ e_{n, 21} & e_{n, 2}\end{array}\right)$. The impact dynamics are

$$
\left\{\begin{array}{l}
m \dot{x}\left(t^{+}\right)=m \dot{x}\left(t^{-}\right) \\
m\left(\dot{y}\left(t^{+}\right)-\dot{y}\left(t^{-}\right)\right)=p_{n, 1}(t)+p_{n, 2}(t) \\
I_{G}\left(\dot{\theta}\left(t^{+}\right)-\dot{\theta}\left(t^{-}\right)\right)=\frac{L}{2}\left(p_{n, 1}(t)-p_{n, 2}(t)\right) \\
\dot{q}_{\mathrm{norm}, 1}\left(t^{+}\right)=-e_{n, 1} \dot{q}_{\mathrm{norm}, 1}\left(t^{-}\right)-e_{n, 12} \dot{q}_{\mathrm{norm}, 2}\left(t^{-}\right) \\
\dot{q}_{\mathrm{norm}, 2}\left(t^{+}\right)=-e_{n, 21} \dot{q}_{\mathrm{norm}, 1}\left(t^{-}\right)-e_{n, 2} \dot{q}_{\mathrm{norm}, 2}\left(t^{-}\right) .
\end{array}\right.
$$

From the fact that $\dot{q}_{\text {norm, } 1}\left(t^{+}\right)=\dot{q}_{\text {norm, } 2}\left(t^{-}\right)=0$, we obtain $\dot{y}\left(t^{-}\right)=\frac{L}{2} \dot{\theta}\left(t^{-}\right)$and $\dot{y}\left(t^{+}\right)=$ $-\frac{L}{2} \dot{\theta}\left(t^{+}\right)$. Since $\dot{q}_{\text {norm, } 1}\left(t^{-}\right)<0$, then $e_{n, 1}=0$. The energetic constraint implies that $e_{n, 21} \in$ $[-1,1]$ and the kinematic constraint $\dot{q}_{\text {norm, } 2}\left(t^{+}\right) \geq 0$ implies $e_{n, 21} \geq 0$, therefore, $e_{n, 21} \in$ $[0,1]$. Recall that $I_{G}=\frac{m}{12}\left(l^{2}+L^{2}\right)$. After some calculations, one gets $p_{n, 1}(t)=-\left(\frac{m L}{4}-\right.$ $\left.\frac{I_{G}}{L}\right) e_{n, 21} \dot{\theta}\left(t^{-}\right)-\frac{m}{2} \dot{y}\left(t^{-}\right)-\frac{I_{G}}{L} \dot{\theta}\left(t^{-}\right)$so $p_{n, 1}(t)=\frac{m}{12}\left[\left(l^{2}-2 L^{2}\right) e_{n, 21}-\left(l^{2}+4 L^{2}\right)\right] \dot{\theta}\left(t^{-}\right)$. Since $\dot{\theta}\left(t^{-}\right)<0$, it follows that $p_{n, 1}(t) \geq 0$ if and only if either $e_{n, 21} \leq \frac{l^{2}+4 L^{2}}{l^{2}-2 L^{2}}$ and $l>\sqrt{2} L$, or $e_{n, 21} \geq 0$ and $l<\sqrt{2} L$. Also, $p_{n, 2}(t)=\frac{m}{12}\left[-\left(4 L^{2}+l^{2}\right) e_{n, 21}-\left(2 L^{2}-l^{2}\right)\right] \dot{\theta}\left(t^{-}\right)$. The kinetic constraint $p_{n, 2}(t) \geq 0$ is equivalent to either $l<\sqrt{2} L$ and $e_{n, 21} \geq 0$, or $l>\sqrt{2} L$ and $e_{n, 21} \geq \frac{l^{2}-2 L^{2}}{4 L^{2}+l^{2}}$. We deduce that the kinematic, kinetic and energetic constraints imply that $e_{n, 21} \in\left[\max \left(0, \frac{l^{2}-2 L^{2}}{l^{2}+4 L^{2}}\right), 1\right]$. This means that rocking is possible in the class of flat rocks (with $0<l<\sqrt{2} L$ ) with $e_{n, 21} \in[0,1]$, and of slender rocks (with $l>\sqrt{2} L$ ) with $e_{n, 21} \geq$ $\frac{l^{2}-2 L^{2}}{4 L^{2}+l^{2}}$. One has $\dot{\theta}\left(t^{+}\right)=e_{n, 21} \dot{\theta}\left(t^{-}\right) \leq 0$ and $\dot{y}\left(t^{+}\right)=-\frac{e_{n, 21} L}{2} \dot{\theta}\left(t^{-}\right) \geq 0$. Notice that in the limit $\frac{l}{L} \rightarrow+\infty$ then $e_{n, 21}=1$ and therefore $\dot{\theta}\left(t^{+}\right)=\dot{\theta}\left(t^{-}\right)$with conservation of energy at the impact: such a model implies that very slender blocks do not lose kinetic energy at the impacts. The admissible domain for $e_{n, 21}$ is depicted in Fig. 2.

Fig. 2 The admissible domain for $e_{n, 21}$ (sliding rocking)

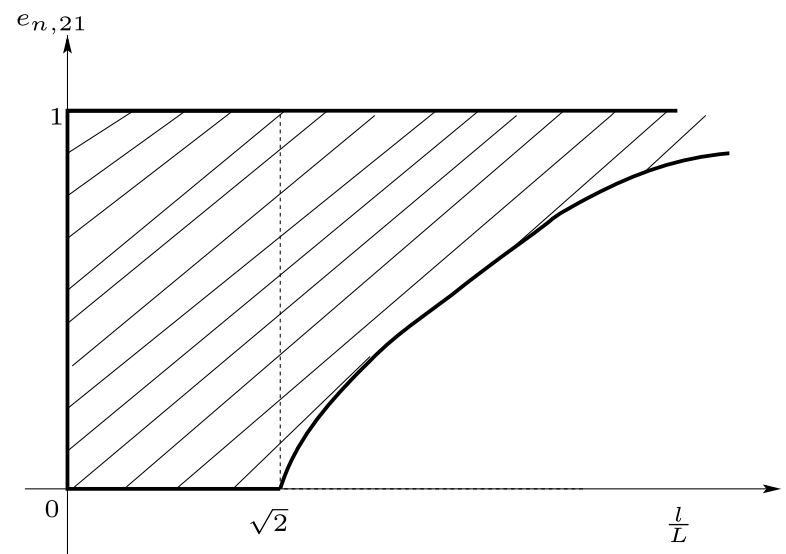


Fig. 3 The admissible domain for $e_{n, 21}$ (sticking rocking)

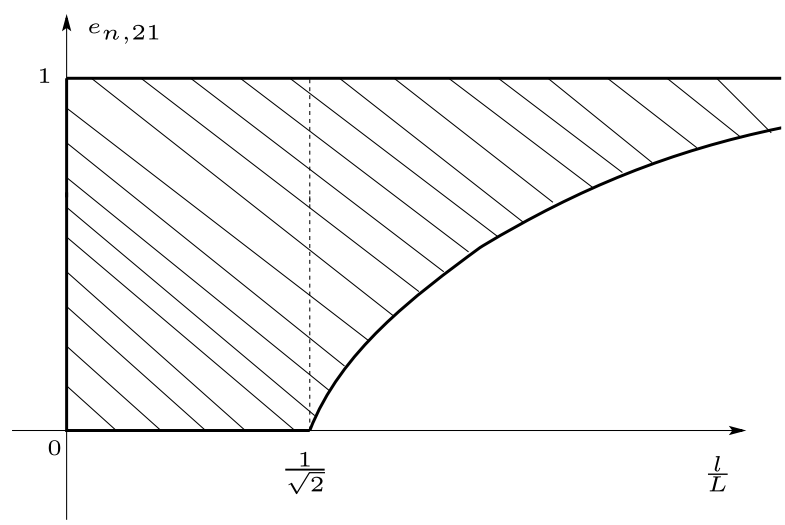

Remark 5 The introduction of coupling terms in $\mathcal{E}_{n}$ allows one to cover a larger set of the rocking motion. Depending on the type of motion (i.e., on the type of kinematic constraint that is imposed on the block), the energetic constraint may imply different bounds on the entries of $\mathcal{E}_{n}$. Indeed the energetic inequality defines a subset in the set of restitution coefficients (the entries of $\mathcal{E}_{n}$ ). The other constraints define sections of this subset.

\subsection{General $\mathcal{E}_{n}, p_{n, 1}(t) \neq 0, p_{n, 2}(t) \neq 0$ (sticking rocking block)}

The kinematic constraints due to the sticking points ( $A$ sticks before the shock, $B$ sticks after it) are given by $\dot{x}\left(t^{-}\right)=-\frac{l}{2} \dot{\theta}\left(t^{-}\right)$and $\dot{q}_{\text {norm, } 2}\left(t^{-}\right)=\dot{y}\left(t^{-}\right)-\frac{L}{2} \dot{\theta}\left(t^{-}\right)=0$. The point $B$ is fixed in both directions after the shock, i.e., $\dot{x}\left(t^{+}\right)=-\frac{l}{2} \dot{\theta}\left(t^{+}\right)$and $\dot{q}_{\text {norm, } 1}\left(t^{+}\right)=\dot{y}\left(t^{+}\right)+$ $\frac{L}{2} \dot{\theta}\left(t^{+}\right)=0$. The impact dynamics is as in (15). It is noteworthy that imposing a priori all the above kinematic constraints, imposes a very particular form of the percussion vector. One obtains $\dot{\theta}\left(t^{+}\right)=e_{n, 21} \dot{\theta}\left(t^{-}\right)$, and since we deal with rocking $e_{n, 21}>0$. Recall also that $e_{n, 1}=$ 0 . The energetic constraint $T\left(t^{+}\right)-T\left(t^{-}\right)=\frac{m\left(l^{2}+L^{2}\right)}{6}\left(e_{n, 21}^{2}-1\right) \dot{\theta}^{2}\left(t^{-}\right) \leq 0$ implies $e_{n, 21} \in$ $[-1,1]$. Therefore, the energetic and kinematic constraints imply $e_{n, 21} \in[0,1]$. One gets $p_{n, 1}(t)=\frac{m}{6 L}\left[\left(2 l^{2}-L^{2}\right) e_{n, 21}-2\left(l^{2}+L^{2}\right)\right] \dot{\theta}\left(t^{-}\right)$, so that $p_{n, 1}(t) \geq 0$ is always satisfied for $e_{n, 21} \in[0,1]$. Now $p_{n, 2}(t)=-\frac{m}{6 L}\left[\left(2\left(l^{2}+L^{2}\right) e_{n, 21}+L^{2}-2 l^{2}\right] \dot{\theta}\left(t^{-}\right)\right.$, from which it follows that $p_{n, 2}(t) \geq 0$ if and only if $e_{n, 21} \in\left[\max \left(0, \frac{2 l^{2}-L^{2}}{2\left(l^{2}+L^{2}\right)}\right), 1\right]$. If $\sqrt{2} l<L$ then $e_{n, 21} \in[0,1]$, and if $\sqrt{2} l>L$ then $e_{n, 21} \geq \frac{2 l^{2}-L^{2}}{2\left(l^{2}+L^{2}\right)}$ that is $e_{n, 21} \in\left[\frac{2 l^{2}-L^{2}}{2\left(l^{2}+L^{2}\right)}, 1\right]$. The admissible domain for $e_{n, 21}$ is depicted in Fig. 3. It is noteworthy that the sticking assumption modifies the admissible domain for $e_{n, 21}$ as can be seen from Figs. 2 and 3. In particular, the critical value $\frac{l}{L}=\sqrt{2}$ for the sliding case is changed to $\frac{1}{\sqrt{2}}$ for the sticking case.

Let us comment that if $\mathcal{E}_{n}$ is diagonal, then one obtains that $e_{n, 1}=0, \dot{q}_{\text {norm, } 2}\left(t^{+}\right)=0$ (since $\dot{q}_{\text {norm }, 2}\left(t^{+}\right)=-e_{n, 2} \dot{q}_{\text {norm }, 2}\left(t^{-}\right)$and $\dot{q}_{\text {norm }, 2}\left(t^{-}\right)=0$ ), so that $\dot{y}\left(t^{+}\right)=\frac{L}{2} \dot{\theta}\left(t^{+}\right)$. This with $\dot{q}_{\text {norm }, 1}\left(t^{+}\right)=\dot{y}\left(t^{+}\right)+\frac{L}{2} \dot{\theta}\left(t^{+}\right)=0$ implies that $\dot{y}\left(t^{+}\right)=\dot{\theta}\left(t^{+}\right)=0$ : only the most dissipative case is handled.

\subsection{Diagonal $\mathcal{E}_{n}, p_{n, 1}(t) \neq 0, p_{n, 2}(t) \neq 0$ (half-rocking block)}

We now turn our attention to the half-rocking motion problem. $A$ stays in contact while $B$ collides the ground and rebounds. The kinematic constraints are $f_{2}(q(t))=0$ and $\dot{y}(t)+$ $\frac{l}{2} \dot{\theta}(t) \sin (\theta(t))-\frac{L}{2} \dot{\theta}(t) \cos (\theta(t))=0$ for all $t$. The post- and pre-impact constraints are 
thus (at a double-impact time one has $\theta(t)=0): \dot{y}\left(t^{+}\right)=\frac{L}{2} \dot{\theta}\left(t^{+}\right)$and $\dot{y}\left(t^{-}\right)=\frac{L}{2} \dot{\theta}\left(t^{-}\right)$. The impact at $B$ means that $\dot{q}_{\text {norm }, 1}\left(t^{+}\right)=-e_{n, 1} \dot{q}_{\text {norm }, 1}\left(t^{-}\right)$with $\dot{q}_{\text {norm }, 1}\left(t^{-}\right)<0$ and $\dot{q}_{\text {norm }, 1}\left(t^{+}\right) \geq$ 0 . Combining all these equalities, we obtain $\dot{\theta}\left(t^{+}\right)=-e_{n, 1} \dot{\theta}\left(t^{-}\right)$and consequently $\dot{y}\left(t^{+}\right)=$ $-e_{n, 1} \dot{y}\left(t^{-}\right)$. Let us now use the impact dynamics in (15). Simple calculations yield:

$$
\left\{\begin{array}{l}
p_{n, 1}(t)=-\frac{m}{12 L}\left(e_{n, 1}+1\right) \dot{\theta}\left(t^{-}\right)\left(4 L^{2}+l^{2}\right) \\
p_{n, 2}(t)=\frac{m}{12 L}\left(e_{n, 1}+1\right) \dot{\theta}\left(t^{-}\right)\left(l^{2}-2 L^{2}\right) .
\end{array}\right.
$$

Therefore, $p_{n, 1}(t) \geq 0$ always, whereas $p_{n, 2}(t) \geq 0 \Leftrightarrow l^{2} \leq 2 L^{2}$. We retrieve again that only flat block $\left(\theta_{12} \leq \frac{\pi}{2}\right)$ can be modeled, as in Sect. 3.2.

The case where one assumes $p_{n, 2}(t)=0$ (the contact force at $A$ remains a function of time) yields from (26) that necessarily $l^{2}=2 L^{2}$ so that only $\theta_{12}=\frac{\pi}{2}$ can be treated. Finally assuming a general restitution matrix $\mathcal{E}_{n}$ as in (25) yields since $\dot{q}_{\text {norm, } 2}\left(t^{-}\right)=0$ and $\dot{q}_{\text {norm }, 2}\left(t^{+}\right)=0$ that $e_{n, 21}=0$, so nothing is brought in the model compared to the diagonal restitution matrix case.

\subsection{Coulomb's friction}

Let us analyze now the collision dynamics when one adds Coulomb's friction at the impulse level and combines it with the generalized restitution rule. The philosophy adopted here is that we force $p_{t, 1}$ and $p_{t, 2}$ to lie inside the friction cone and examine the consequences on the constraints to be imposed on the model parameters (restitution and friction coefficients). This may be seen as an extension to the block/ground system of the results in $[35,53]$ that concern the study of 1-impacts with Coulomb's friction and its relationship with tangential restitution.

\subsubsection{Diagonal $\mathcal{E}_{n}, p_{n, 1}(t) \neq 0, p_{n, 2}(t) \neq 0$ (rocking block)}

Coulomb's friction is introduced at $A$ and $B$, by setting at each of these point as

$$
p_{t, i}(t) \in-\mu p_{n, i}(t) \operatorname{sgn}\left(v_{t, i}\left(t^{+}\right)\right)
$$

where $v_{t, 1}=\dot{x}+\frac{\dot{\theta}}{2}(l \cos (\theta)-L \sin (\theta)), v_{t, 2}=\dot{x}+\frac{\dot{\theta}}{2}(l \cos (\theta)+L \sin (\theta))$ are the horizontal velocities at $B$ and $A$, respectively, $\mu \geq 0$ is a friction coefficient, $\operatorname{sgn}(\cdot)$ is the multivalued at zero signum function, i.e., $\operatorname{sgn}(0)=[-1,1]$. It is common in impact mechanics to introduce Coulomb's friction at the impulse level, but this may yield an energetically inconsistent model when coupled with a kinematic restitution coefficient and when the tangential velocity reverses its sign during the impact $[8,13,50]$. Notice also that the argument is the right limit of the tangential velocity, hence allowing for a change of sign during the impact. Introducing $\lambda_{t, 1}$ and $\lambda_{t, 2}$, the tangential reactions at $B$ and $A$, respectively, and the corresponding tangential impulses $p_{t, 1}$ and $p_{t, 2}$, the impact dynamics become

$$
\left\{\begin{array}{l}
m\left(\dot{x}\left(t^{+}\right)-\dot{x}\left(t^{-}\right)\right)=p_{t, 1}(t)+p_{t, 2}(t) \\
m\left(\dot{y}\left(t^{+}\right)-\dot{y}\left(t^{-}\right)\right)=p_{n, 1}(t)+p_{n, 2}(t) \\
I_{G}\left(\dot{\theta}\left(t^{+}\right)-\dot{\theta}\left(t^{-}\right)\right)=\frac{L}{2}\left(p_{n, 1}(t)-p_{n, 2}(t)\right)+\frac{l}{2}\left(p_{t, 1}(t)+p_{t, 2}(t)\right) \\
p_{t, i}(t) \in-\mu p_{n, i}(t) \operatorname{sgn}\left(v_{t, i}\left(t^{+}\right)\right), \quad i=1,2 \\
\dot{q}_{\text {norm }, 1}\left(t^{+}\right)=-e_{n, 1} \dot{q}_{\text {norm }, 1}\left(t^{-}\right) \\
\dot{q}_{\text {norm }, 2}\left(t^{+}\right)=-e_{n, 2} \dot{q}_{\text {norm }, 2}\left(t^{-}\right) .
\end{array}\right.
$$


Suppose that the block undergoes perfect rocking motion. Before the impact $A$ is sticking, so $\dot{q}_{\text {norm }, 2}\left(t^{-}\right)=0$ and $\dot{q}_{\text {norm }, 2}\left(t^{+}\right)=0$. Thus $\dot{y}\left(t^{-}\right)=\frac{L}{2} \dot{\theta}\left(t^{-}\right)$and $\dot{y}\left(t^{+}\right)=\frac{L}{2} \dot{\theta}\left(t^{+}\right)$. Therefore rocking is still impossible because the friction cannot help in making $A$ detach from the ground after the shock. At best, this model may describe half-rocking motion. Moreover, $\dot{q}_{\text {norm }, 1}\left(t^{+}\right)=0$ is equivalent to $\dot{y}\left(t^{+}\right)=-\frac{L}{2} \dot{\theta}\left(t^{+}\right)$, so $\dot{q}_{\text {norm }, 2}\left(t^{+}\right)=\dot{q}_{\text {norm }, 1}\left(t^{+}\right)=$ 0 implies that $\dot{\theta}\left(t^{+}\right)=0$ (and $\dot{y}\left(t^{+}\right)=0$ ): only the most dissipative case can be handled. Notice that at a 2-impact one has $\theta(t)=0$, therefore, $v_{t, 1}=v_{t, 2}=\dot{x}+\frac{l}{2} \dot{\theta}$. Sticking rocking implies that $\dot{x}\left(t^{-}\right)=-\frac{l}{2} \dot{\theta}\left(t^{-}\right)$and $\dot{x}\left(t^{+}\right)=-\frac{l}{2} \dot{\theta}\left(t^{+}\right)$; see Sect. 3.4. Thus, imposing tangential restitution of the form $v_{t, 1}\left(t^{+}\right)=e_{t, 1} v_{t, 1}\left(t^{-}\right), v_{t, 2}\left(t^{+}\right)=e_{t, 2} v_{t, 2}\left(t^{-}\right)$, first boils down to defining a unique coefficient $e_{t}$ since both velocities are equal at 2-impacts, second is meaningless since the kinematic constraints transform these two tangential impact laws into $0=e_{t} 0$. Off-diagonal terms in $\mathcal{E}_{n}$ are necessary to allow for rocking motion and to define tangential restitution; see Sect. 6 and (55). They correspond to the "distance" effects which are known to be necessary to span the whole subspace of admissible post-impact velocities in chains of balls [20]; see Sect. 7.

\subsubsection{General $\mathcal{E}_{n}, p_{n, 1}(t) \neq 0, p_{n, 2}(t) \neq 0$ (sticking rocking motion)}

Let us consider (28) with off-diagonal terms in $\mathcal{E}_{n}$, i.e., we consider since $\dot{q}_{\text {norm, } 2}\left(t^{+}\right)=0$ that $\dot{q}_{\text {norm }, 1}\left(t^{+}\right)=-e_{n, 1} \dot{q}_{\text {norm }, 1}\left(t^{-}\right)$and $\dot{q}_{\text {norm }, 2}\left(t^{+}\right)=-e_{n, 21} \dot{q}_{\text {norm }, 1}\left(t^{-}\right)$. The perfect rocking assumption implies sticking of the contact points in both normal and tangential directions, and we obtain as above $\dot{y}\left(t^{-}\right)=\frac{L}{2} \dot{\theta}\left(t^{-}\right), \dot{y}\left(t^{+}\right)=-\frac{L}{2} \dot{\theta}\left(t^{+}\right), \dot{x}\left(t^{-}\right)=-\frac{l}{2} \dot{\theta}\left(t^{-}\right), \dot{x}\left(t^{+}\right)=$ $-\frac{l}{2} \dot{\theta}\left(t^{+}\right), e_{n, 1}=0$. Combining this with the restitution rule yields $\dot{\theta}\left(t^{+}\right)=e_{n, 21} \dot{\theta}\left(t^{-}\right)$. It follows that the energetic constraint implies that $e_{n, 21} \in[-1,1]$. Let us recall that when sticking occurs in the tangential direction, the model says that $p_{t, i}(t) \in-\mu p_{n, i}(t)[-1,1]=$ $\left[-\mu p_{n, i}, \mu p_{n, i}\right]$. Equivalently, there exists $\xi_{i}(t) \in\left[-\mu p_{n, i}(t), \mu p_{n, i}(t)\right]$ such that $p_{t, i}(t)=$ $\xi_{i}(t), i=1,2$. Taking into account all these constraints and the dynamics in (28), one calculates that

$$
\left\{\begin{array}{l}
p_{n, 1}(t)=\frac{m}{6 L}\left[\left(2 l^{2}-L^{2}\right) e_{n, 21}-2\left(l^{2}+L^{2}\right)\right] \dot{\theta}\left(t^{-}\right) \\
p_{n, 2}(t)=-\frac{m}{6 L}\left[\left(2 l^{2}+2 L^{2}\right) e_{n, 21}+L^{2}-2 l^{2}\right] \dot{\theta}\left(t^{-}\right) .
\end{array}\right.
$$

It follows that $p_{n, 1}(t) \geq 0$ for all $e_{n, 21} \in[0,1]$, while $p_{n, 2}(t) \geq 0$ if and only if $e_{n, 21} \in$ $\left[\max \left(0, \frac{2 l^{2}-L^{2}}{2 l^{2}+2 L^{2}}\right), 1\right]$. The Coulomb's friction adds another constraint. Indeed from the first equation in (28) and the above equalities, one obtains $\frac{m l}{2}\left(-\dot{\theta}\left(t^{+}\right)+\dot{\theta}\left(t^{-}\right)\right)=\xi_{1}(t)+\xi_{2}(t)$, from which it follows that

$$
\frac{l}{L} \frac{e_{n, 21}-1}{e_{n, 21}+1} \in[-\mu, \mu]
$$

The inclusion (30) constrains the aspect ratio, the coefficient of friction and $e_{n, 21}$. The dependency between $e_{n, 21}$ and $\mu$ is expected since $e_{n, 21}$ may be seen as a macroscopic coefficient modeling some tangential effects.

\subsubsection{General $\mathcal{E}_{n}, p_{n, 1}(t) \neq 0, p_{n, 2}(t) \neq 0$ (sliding rocking motion)}

Let us consider the same assumptions, however this time the contact points are supposed to slip so that $p_{t, i}= \pm \mu p_{n, i}$, the sign depending on the relative slip direction. Assume first that slip occurs to the right at the impact, that is, $v_{t, i}\left(t^{+}\right)>0, i=1,2$. One obtains

$$
\left\{\begin{array}{l}
p_{n, 1}(t)=\frac{m}{24 L}\left[\left(l^{2}-2 L^{2}-3 \mu l L\right) e_{n, 21}-\left(l^{2}+4 L^{2}+3 \mu l L\right)\right] \dot{\theta}\left(t^{-}\right) \\
p_{n, 2}(t)=-\frac{m}{12 L}\left[\left(3 \mu l L-l^{2}-4 L^{2}\right) e_{n, 21}+3 \mu l L-2 L^{2}+l^{2}\right] \dot{\theta}\left(t^{-}\right) .
\end{array}\right.
$$


It follows that $p_{n, 1}(t) \geq 0$ for all $e_{n, 21} \in[0,1]$, while $p_{n, 2}(t) \geq 0$ if and only if $e_{n, 21} \in$ $\left[\max \left(0, e_{c, 1}^{+}\right), 1\right]$ with $e_{c, 1}^{+}(\mu, L, l) \triangleq \frac{l^{2}-2 L^{2}+3 \mu l L}{4 L^{2}+l^{2}-3 \mu l L}$. Using the first equation in (28) and (14) and (18), one finds that

$$
\left(\mathcal{E}_{\tan }+1\right) \dot{q}_{\tan }\left(t^{-}\right)=-\frac{\mu \sqrt{m} L}{2}\left(1+e_{n, 21}\right) \dot{\theta}\left(t^{-}\right) .
$$

This shows that Coulomb's friction and the off-diagonal term in $\mathcal{E}_{n}$ imply a generalized tangential restitution coefficient that is not constant but depends on the preimpact conditions. Suppose now that slip occurs to the left at the impact, that is, $v_{t, i}\left(t^{+}\right)<0, i=1,2$ (remind that we are still studying the case when $B$ collides the ground while the block rotates around $A)$. One logically obtains

$$
\left\{\begin{array}{l}
p_{n, 1}(t)=\frac{m}{24 L}\left[\left(l^{2}-2 L^{2}+3 \mu l L\right) e_{n, 21}-\left(l^{2}+4 L^{2}-3 \mu l L\right)\right] \dot{\theta}\left(t^{-}\right) \\
p_{n, 2}(t)=-\frac{m}{12 L}\left[\left(-3 \mu l L-l^{2}-4 L^{2}\right) e_{n, 21}-3 \mu l L-2 L^{2}+l^{2}\right] \dot{\theta}\left(t^{-}\right) .
\end{array}\right.
$$

We infer that the kinetic and kinematic constraints imply $e_{n, 21} \in\left[\max \left(0, e_{c, 1}^{-}\right), 1\right]$ with $e_{c, 1}^{-}(\mu, L, l) \triangleq \frac{l^{2}-2 L^{2}-3 \mu l L}{4 L^{2}+l^{2}+3 \mu l L}$, and

$$
\left(\mathcal{E}_{\tan }+1\right) \dot{q}_{\tan }\left(t^{-}\right)=\frac{\mu \sqrt{m} L}{2}\left(1+e_{n, 21}\right) \dot{\theta}\left(t^{-}\right) .
$$

The loss of kinetic energy provides the following inequality, depending on left $\left(v_{t, i}\left(t^{+}\right)<\right.$ 0 and minus sign in (35)) or right $\left(v_{t, i}\left(t^{+}\right)>0\right.$ and plus sign in (35)) sliding:

$$
\left(\mu^{2} L^{2}\left(1+e_{n, 21}\right)^{2}+\frac{4 L^{2}+l^{2}}{6}\left(e_{n, 21}^{2}-1\right)\right) \dot{\theta}\left(t^{-}\right) \pm 4 \mu L\left(1+e_{n, 21}\right) \dot{x}\left(t^{-}\right) \geq 0
$$

where we used that the first and second equalities in (28) yield $\dot{x}\left(t^{+}\right)-\dot{x}\left(t^{-}\right)=\frac{\mu L}{2}(1+$ $\left.e_{n, 21}\right) \dot{\theta}\left(t^{-}\right)$if $v_{t, i}\left(t^{+}\right)>0$ and $\dot{x}\left(t^{+}\right)-\dot{x}\left(t^{-}\right)=-\frac{\mu L}{2}\left(1+e_{n, 21}\right) \dot{\theta}\left(t^{-}\right)$if $v_{t, i}\left(t^{+}\right)<0$, and the fact that $\dot{\theta}\left(t^{-}\right)<0$. When $\mu=0$, one recovers that $e_{n, 21} \in[-1,1]$. Clearly, the energetic constraint depends on the preimpact velocities, that is, of the impact dynamics initial data. We may nevertheless state the following result. Assume that $v_{t, i}\left(t^{+}\right)>0$ and $v_{t, i}\left(t^{-}\right)>0$. Thus, $\dot{x}\left(t^{-}\right)>-\frac{l}{2} \dot{\theta}\left(t^{-}\right)>0$. The analysis of the expression between brackets in the lefthand side of (35) reveals that if $\mu \leq \mu_{c} \triangleq \sqrt{\frac{4 L^{2}+l^{2}}{6 L^{2}}}$ then the first term in the left-hand side of (35) is nonnegative if and only if $e_{n, 21} \leq e_{c, 2}^{+} \triangleq-\frac{2 \mu^{2} L^{2}-\frac{4 L^{2}+l^{2}}{3}}{\frac{\mu^{2} L^{2}}{2}+\frac{4 L^{2}+l^{2}}{3}}$, where $e_{c, 2}^{+}(\mu>0, L, l)<1$ and $e_{c, 2}^{+}(0, L, l)=1$. If $\mu>\mu_{c}$, then there is no nonnegative value of $e_{n, 21}$ such that the first term in the left-hand side of (35) is nonnegative. We infer that a sufficient condition such that (35) is satisfied (equivalently the energetical constraint is satisfied) when there is no tangential velocity reversal with $v_{t, i}\left(t^{+}\right)>0, v_{t, i}\left(t^{-}\right)>0$, is that $\mu \leq \mu_{c}$ and $e_{n, 21} \leq e_{c, 2}^{+}$. Thus, the kinetic, kinematic, and energetic constraints are satisfied provided that $e_{n, 21} \in$ $\left[\max \left(0, e_{c, 1}^{+}(\mu, L, l)\right), \min \left(1, e_{c, 2}^{+}(\mu, L, l)\right)\right]$ and $e_{c, 2}^{+}(\mu, L, l) \geq e_{c, 1}^{+}(\mu, L, l)$.

We do not continue the calculations that would allow us to give a complete characterization of the inequality (35), because on one hand this would take lengthy developments, on the other hand our goal is mainly to show that coupling kinematic restitution and Coulomb's friction at the impulse level, leads to a complex set of constraints that have to be satisfied by the rather simple coefficients of restitution. Even in the simpler case of 1-impacts between 
rough bodies, it is well known that the kinetic energy constraint yields somewhat complex expressions from which critical values of the coefficients may be derived, see [6,7] [8, pp. 329-331]. The above analysis may be seen as an extension toward 2-impacts.

\subsubsection{General $\mathcal{E}_{n}, p_{n, 1}(t) \neq 0, p_{n, 2}(t) \neq 0$ (sliding half-rocking motion)}

In this case necessarily $e_{n, 21}=0$ and the value $e_{n, 12}$ plays no role since $\dot{q}_{\text {norm, } 2}\left(t^{-}\right)=0$. So, adding off-diagonal terms in $\mathcal{E}_{n}$ is meaningless. One has $\dot{q}_{\text {norm }, 1}\left(t^{+}\right)=-e_{n, 1} \dot{q}_{\text {norm }, 1}\left(t^{-}\right)$ so that $e_{n, 1}>0$, and half-rocking implies that $\dot{\theta}\left(t^{+}\right)>0$ while $\dot{\theta}\left(t^{-}\right)<0$. One has $\dot{q}_{\text {norm }, 2}\left(t^{-}\right)=\dot{q}_{\text {norm }, 2}\left(t^{+}\right)=0$ so that $\dot{y}\left(t^{+}\right)=\frac{L}{2} \dot{\theta}\left(t^{+}\right)$and $\dot{y}\left(t^{-}\right)=\frac{L}{2} \dot{\theta}\left(t^{-}\right)$, and as a consequence $\dot{q}_{\text {norm }, 1}\left(t^{+}\right)=-e_{n, 1} \dot{q}_{\text {norm }, 1}\left(t^{-}\right)$implies $\dot{\theta}\left(t^{+}\right)=-e_{n, 1} \dot{\theta}\left(t^{-}\right)$. Suppose first that $v_{t, i}\left(t^{+}\right)>0$. The impact dynamics in (28) yield

$$
\left\{\begin{array}{l}
p_{n, 1}(t)=-\frac{1}{L}\left(I_{G}+\frac{L+\mu l}{2} \frac{m L}{2}\right)\left(e_{n, 1}+1\right) \dot{\theta}\left(t^{-}\right) \\
p_{n, 2}(t)=\frac{m}{12 L}\left(e_{n, 1}+1\right)\left(l^{2}-2 L^{2}+3 \mu l L\right) \dot{\theta}\left(t^{-}\right)
\end{array}\right.
$$

We have that $p_{n, 1}(t) \geq 0$ for all $e_{n, 1} \geq 0$, and $p_{n, 2}(t) \geq 0$ if and only if $\mu \leq \frac{2 L^{2}-l^{2}}{3 l L}$ when $e_{n, 1} \geq 0$. We deduce that necessarily $l \leq \sqrt{2} L$ so that only flat blocks can be handled. Let us now examine the kinetic energy loss at the impact time. Using all the above equalities, the kinetic constraint is given by

$$
\left(\frac{\mu^{2} L^{2}}{4}\left(e_{n, 1}+1\right)^{2}+\left(e_{n, 1}^{2}-1\right)\right) \dot{\theta}\left(t^{-}\right)+L\left(e_{n, 1}+1\right) \dot{x}\left(t^{-}\right) \geq 0 .
$$

Let us study the case where there is no velocity reversal, that is, $v_{t, i}\left(t^{-}\right)>0$, which implies that $\dot{x}\left(t^{-}\right)>-\frac{l}{2} \dot{\theta}\left(t^{-}\right)>0$. Thus, a sufficient condition for (37) to be satisfied is that:

$$
\left(3 \mu^{2} L^{2}+l^{2}+4 L^{2}\right) e_{n, 1}^{2}+6 \mu^{2} L^{2} e_{n, 1}+3 \mu^{2} L^{2}-l^{2}-4 L^{2} \leq 0 .
$$

This inequality is in turn satisfied if and only if $e_{n, 1} \in\left[0, e_{c, 3}^{+}\right]$, with $e_{c, 3}^{+}(l, L, \mu) \triangleq$ $\frac{l^{2}+4 L^{2}-3 \mu^{2} L^{2}}{l^{2}+4 L^{2}+3 \mu^{2} L^{2}}$. One has $e_{c, 3}^{+}(l, L, \mu) \geq 0$ if and only if $\mu \leq \sqrt{\frac{l^{2}+4 L^{2}}{3 L^{2}}}$. To summarize the kinetic, kinematic, and energetic constraints imply when $v_{t, i}\left(t^{+}\right)>0$ and $v_{t, i}\left(t^{-}\right)>0$ :

$$
e_{n, 21}=0, \quad e_{n, 1} \in\left[0, e_{c, 3}^{+}(l, L, \mu)\right], \quad \mu \leq \min \left(\frac{2 L^{2}-l^{2}}{3 l L}, \sqrt{\frac{l^{2}+4 L^{2}}{3 L^{2}}}\right), \quad l \leq \sqrt{2} L .
$$

When $\mu=0$, then $e_{c, 3}^{+}=1$ and one recovers the results of Sect. 3.5. When there is a tangential velocity reversal, then $v_{t, i}\left(t^{-}\right)<0$ and one cannot conclude about the sign of $\dot{x}\left(t^{-}\right)$. So, the energetic constraint can hardly be stated independently of the preimpact velocities. The case $v_{t, i}\left(t^{+}\right)<0$ can be led in a similar way, and is not reported here for the sake of briefness.

\subsubsection{Conclusions}

The conclusion to be drawn from this section is that the introduction of Coulomb's friction at the impulse level when coupled to kinematic restitution laws, yields an impact dynamics that may be overconstrained and the coefficients should satisfy conditions whose mechanical interpretation is not obvious. When using such kinematic laws, one may better remain at a more simple modeling level, using only the matrix $\mathcal{E}$. 
Remark 6 The impact law with friction studied and used in [12], inspired from [44], and which is in fact Moreau's impact law (with $\mathcal{E}_{n}=\operatorname{diag}(e)$ ) with Coulomb friction at the impulse level, is therefore unable to reach the rocking motion within the subspace of admissible post-impact velocities. A similar model with $\mathcal{E}_{n}=\operatorname{diag}\left(e_{n, i}\right)$ and Coulomb friction at the impulse level is also used in [5, 16-18]. Same conclusions hold for impact laws with friction as studied in [49], among others. It is noteworthy that the combination of Newton's law in the normal direction and of Coulomb friction at the impulse level in the (local) tangential direction, can yield energetical inconsistencies (gain of kinetic energy at the collision) [8, 13]. General conditions that prevent such behaviors are stated in [29]. In [37, 38], an approach similar to the above one using the kinetic metric is chosen, however, no tangential effects are considered. Other works may be found in [11, 25], however, they use compliant (spring,dashpot)-like contact/impact models and are consequently conceptually outside the scope of rigid body restitution impact rules, which are of interest in this work.

\subsection{The angular velocity restitution law}

Let us now start from the angular restitution law that is widely used in the earthquake engineering literature:

$$
\dot{\theta}\left(t^{+}\right)=-r \dot{\theta}\left(t^{-}\right)
$$

(without considering it as a consequence of the above generalized kinematic law) and examine the consequences of rocking motion. First of all, it has to be remarked that the kinematic restitution law in (40) is not associated with the unilateral constraints in (1) (or equivalently (2)), except if $y$ is a constant in which case it makes perfect mechanical sense because $\nabla f_{i}^{T}(q) \dot{q}=\dot{\theta}$. However, in general, $y$ is not constant and one may suspect that the impact law (40) stems from another constraint (see Sect. 6). Let us examine this in more detail.

\subsubsection{Sliding rocking block}

Let us consider first the rocking case (ii) above: the block rotates around $A$ with $\dot{\theta}<0$, then hits the ground at $B$ and starts rotating around $B$ with $\dot{\theta}<0$ while $A$ detaches from the constraint boundary. From (15) we deduce at the impact time $(\theta(t)=0)$ :

$$
\left\{\begin{array}{l}
m\left(\dot{y}\left(t^{+}\right)-\dot{y}\left(t^{-}\right)\right)=p_{n, 1}+p_{n, 2} \\
I_{G}\left(\dot{\theta}\left(t^{+}\right)-\dot{\theta}\left(t^{-}\right)\right)=\frac{L}{2}\left(p_{n, 1}-p_{n, 2}\right) \\
\dot{q}_{\text {norm }, 1}\left(t^{+}\right)=\dot{y}\left(t^{+}\right)+\frac{L}{2} \dot{\theta}\left(t^{+}\right)=0 \\
\dot{q}_{\text {norm }, 2}\left(t^{-}\right)=\dot{y}\left(t^{-}\right)-\frac{L}{2} \dot{\theta}\left(t^{-}\right)=0 .
\end{array}\right.
$$

The loss of kinetic energy at the impact is found to be equal to $T_{L}(t)=\frac{1}{2}\left(\frac{m L^{2}}{4}+I_{G}\right)\left(r^{2}-\right.$ 1) $\dot{\theta}^{2}\left(t^{-}\right)$so that $r \in[-1,1]$ from the energetic constraint. Since we examine the rocking motion we have $\operatorname{sgn}\left(\dot{\theta}\left(t^{-}\right)\right)=\operatorname{sgn}\left(\dot{\theta}\left(t^{+}\right)\right)$so that $r \leq 0$, hence $r \in[-1,0]$. From (41), one computes that

$$
\left\{\begin{array}{l}
p_{n, 1}(t)=\left(\frac{m L}{4}-\frac{2 I_{G}}{L}\right) r-\frac{I_{G}}{L} \dot{\theta}\left(t^{-}\right)-\frac{m}{2} \dot{y}\left(t^{-}\right) \\
p_{n, 2}(t)=\left(\frac{m L}{4}+\frac{2 I_{G}}{L}\right) r+\frac{I_{G}}{L} \dot{\theta}\left(t^{-}\right)-\frac{m}{2} \dot{y}\left(t^{-}\right) .
\end{array}\right.
$$


Fig. 4 The admissible domain for $r$ (sliding rocking motion)

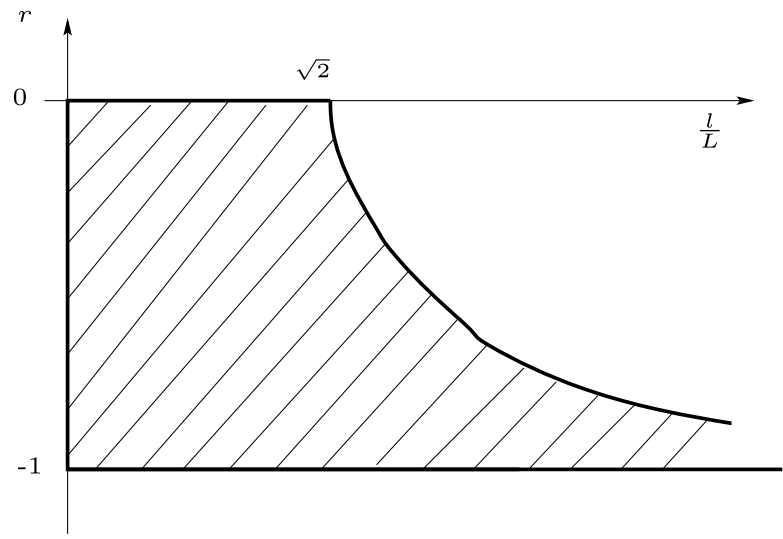

In order to respect the kinetic constraint $p_{n, 1}(t) \geq 0$, one has to have from (42) (recall that $\left.I_{G}=\frac{m}{12}\left(l^{2}+L^{2}\right)\right)$ :

$$
r \leq \frac{l^{2}+L^{2}+6 L \frac{\dot{y}\left(t^{-}\right)}{\dot{\theta}\left(t^{-}\right)}}{2 L^{2}-l^{2}}=\frac{l^{2}+4 L^{2}}{2 L^{2}-l^{2}}(>0)
$$

if $2 L^{2}-l^{2}>0$, and

$$
r \geq \frac{l^{2}+L^{2}+6 L \frac{\dot{y}\left(t^{-}\right)}{\dot{\theta}\left(t^{-}\right)}}{2 L^{2}-l^{2}}=\frac{l^{2}+4 L^{2}}{2 L^{2}-l^{2}}(<0)
$$

if $2 L^{2}-l^{2}<0$ (slender block), and recalling that $\dot{\theta}\left(t^{-}\right)<0, \dot{y}\left(t^{-}\right)<0, \dot{y}\left(t^{-}\right)=\frac{L}{2} \dot{\theta}\left(t^{-}\right)$. Both these upper-bounds are respected for $r \in[-1,0]$, so that the first kinetic constraint is satisfied for $r \in[-1,0]$. Let us now examine $p_{n, 2}(t) \geq 0$. From (42) one has $p_{n, 2}(t)=$ $m\left(\frac{\left(4 L^{2}+l^{2}\right) r-\left(2 L^{2}-l^{2}\right)}{12 L}\right) \dot{\theta}\left(t^{-}\right)$. It follows that $p_{n, 2}(t) \geq 0 \Leftrightarrow l \leq \sqrt{2} L$ and $r \in[-1,0]$, or $l>$ $\sqrt{2} L$ and $|r| \geq \frac{l^{2}-2 L^{2}}{4 L^{2}+l^{2}}$. Notice that $\frac{-l^{2}+2 L^{2}}{4 L^{2}+l^{2}} \geq-1$ that is equivalent to $-l^{2}+2 L^{2} \geq-4 L^{2}-l^{2}$ which is satisfied for all aspect ratios $\frac{l}{L}$. We conclude that $r \in\left[-1, \min \left(0, \frac{l^{2}-2 L^{2}}{4 L^{2}+l^{2}}\right)\right]$. In Fig. 4 is depicted $r$ as a function of $\frac{l}{L}$. Notice that $\dot{q}_{\text {norm }, 2}\left(t^{+}\right)=r \dot{q}_{\text {norm }, 1}\left(t^{-}\right)$, so $r=-e_{n, 21}$ : the angular coefficient of restitution adds a coupling in the matrix $\mathcal{E}_{n}$. It is interesting to see that very slender blocks with large aspect ratio $\frac{l}{L}$ tend to lose less energy at the impact, according to this impact model.

Finally, it is easily computed that if $p_{n, 2}(t)=0$ then $p_{n, 1}(t)=\frac{m L}{2}(r-1) \dot{\theta}\left(t^{-}\right)=$ $-\frac{2 I_{G}}{L}(r+1) \dot{\theta}\left(t^{-}\right)$, which yields $r=\frac{2 L^{2}-l^{2}}{4 L^{2}+l^{2}}$. We recover here the value in (23) that was obtained with the generalized kinematic law, and with the assumption that $p_{n, 2}(t)=0$. Therefore, if $l<\sqrt{2} L$, then $r>0$ and $\operatorname{sgn}\left(\dot{\theta}\left(t^{+}\right)\right)=-\operatorname{sgn}\left(\dot{\theta}\left(t^{-}\right)\right)$. It is not possible that the block sticks at $B$ after the shock for rocking. Thus, with these assumptions, rocking cannot be modeled if $l \leq \sqrt{2} L$. If $r=\sqrt{2} L$ then $r=0$ and the block stops after the shock since $\dot{\theta}\left(t^{+}\right)=0$. Finally, if $l>\sqrt{2} L$ then $r<0$ and $\operatorname{sgn}\left(\dot{\theta}\left(t^{+}\right)\right)=\operatorname{sgn}\left(\dot{\theta}\left(t^{-}\right)\right)=-1$. The block can stick at $B$ in the normal direction and continue to rock. One obtains $\dot{y}\left(t^{+}\right)=\frac{L}{2} \frac{2 L^{2}-l^{2}}{4 L^{2}+l^{2}} \dot{\theta}\left(t^{-}\right)>0$. The energetic constraint implies that $r \in[-1,1]$, and together with the kinematic constraint for rocking one has $r \in[-1,0)$. This is satisfied by $r=\frac{2 L^{2}-l^{2}}{4 L^{2}+l^{2}}$. 


\subsubsection{Sticking free-rocking block}

In many papers, $r$ is calculated by equaling the moment of momentum at the rotating point, before and after the impact. Then one finds $r_{\mathrm{H}}=-\frac{2 l^{2}-L^{2}}{2 L^{2}+2 l^{2}}=-\left(1-\frac{3}{2} \sin ^{2}(\alpha)\right)[27,30]$, where $\alpha$ is as in Fig. 1. We may name this $r_{\mathrm{H}}$ the Housner angular velocity restitution coefficient, since it was introduced in [27]. Notice that $r_{\mathrm{H}}\left(\frac{l}{L}\right)$ varies between $\frac{1}{2}$ for $\frac{l}{L}=0$ to -1 for $\frac{l}{L}=+\infty$, and is zero for $\frac{l}{L}=\frac{1}{\sqrt{2}}$. If we suppose that the problem is frictionless, then $\dot{x}=0$ and $x$ is constant for zero initial data. The moment of momentum at $B$ when free rocking occurs with rotation around $A$ and collision at $B$, is equal to

$$
\sigma_{B}=\left(\begin{array}{c}
0 \\
0 \\
I_{G} \dot{\theta}
\end{array}\right)+m\left(\begin{array}{c}
-\frac{L}{2} \\
\frac{l}{2} \\
0
\end{array}\right) \times\left(\begin{array}{l}
\dot{x} \\
\dot{y} \\
0
\end{array}\right)
$$

so $\sigma_{B}=I_{G} \dot{\theta}-m \frac{L}{2} \dot{y}-m \frac{l}{2} \dot{x}$. Thus, taking into account the kinematic constraints $\dot{y}\left(t^{-}\right)=$ $\frac{L}{2} \dot{\theta}\left(t^{-}\right)$and $\dot{y}\left(t^{+}\right)=-\frac{L}{2} \dot{\theta}\left(t^{+}\right)$, one finds that $\sigma_{B}\left(t^{+}\right)=\sigma_{B}\left(t^{-}\right)$gives $r=\frac{2 L^{2}-l^{2}}{4 L^{2}+l^{2}}$. This indeed agrees with (23), but not with the above value $r_{\mathrm{H}}=-\frac{2 l^{2}-L^{2}}{2 L^{2}+2 l^{2}}$ that is used in the literature, which is found by assuming another kinematic constraint: the contact points do not slip. This is in fact a very approximate way of introducing friction that is responsible for stick/slip behavior, and may explain why this $r$ is often found not to match with the one from experimental results.

Let us now analyze the free-rocking motion where the contact points stick. The kinematic constraints are as in Sect. 3.4: $\dot{x}\left(t^{-}\right)=-\frac{l}{2} \dot{\theta}\left(t^{-}\right), \dot{y}\left(t^{-}\right)-\frac{L}{2} \dot{\theta}\left(t^{-}\right)=0, \dot{x}\left(t^{+}\right)=-\frac{l}{2} \dot{\theta}\left(t^{+}\right)$and $\dot{y}\left(t^{+}\right)+\frac{L}{2} \dot{\theta}\left(t^{+}\right)=0$. From (15), the impact dynamics are given by

$$
\left\{\begin{array}{l}
m\left(\dot{x}\left(t^{+}\right)-\dot{x}\left(t^{-}\right)\right)=\frac{m l L}{4 I_{G}+m l^{2}}\left(p_{n, 2}(t)-p_{n, 1}(t)\right)=p_{t, 1}(t)+p_{t, 2}(t) \\
m\left(\dot{y}\left(t^{+}\right)-\dot{y}\left(t^{-}\right)\right)=p_{n, 1}(t)+p_{n, 2}(t) \\
I_{G}\left(\dot{\theta}\left(t^{+}\right)-\dot{\theta}\left(t^{-}\right)\right)=\frac{L}{2}\left(p_{n, 1}(t)-p_{n, 2}(t)\right)+\frac{l}{2}\left(p_{t, 1}+p_{t, 2}\right) \\
\dot{\theta}\left(t^{+}\right)=-r \dot{\theta}\left(t^{-}\right) .
\end{array}\right.
$$

One has $T_{L}(t)=T\left(t^{+}\right)-T\left(t^{-}\right)=\left(\frac{m}{8}\left(l^{2}+L^{2}\right)+\frac{I_{G}}{2}\right)\left(r^{2}-1\right) \dot{\theta}^{2}\left(t^{-}\right)$so that $T_{L}\left(t^{-}\right) \leq 0 \Leftrightarrow$ $r \in[-1,1]$. Note at once that such an $r$ satisfies the energetic constraint $r \in[-1,1]$, and from the kinematic constraint that both $\dot{\theta}\left({ }^{-}\right)$and $\dot{\theta}\left({ }^{+}\right)$have the same sign, i.e., $r<0$. Thus, the energetic and kinematic constraints imply that $r \in[-1,0]$. One finds after calculations $p_{n, 1}(t)=\left(\frac{m L}{4}(r-1)-\frac{4 I_{G}+m l^{2}}{4 L}\right) \dot{\theta}\left(t^{-}\right)$, and $p_{n, 2}(t)=\left(\frac{m L}{4}(r-1)+\frac{4 I_{G}+m l^{2}}{4 L}(r+1)\right) \dot{\theta}\left(t^{-}\right)$. Therefore, $p_{n, 1}(t) \geq 0$ since $r \in[-1,0]$, and $p_{n, 2}(t) \geq 0$ if and only if $r \leq \frac{L^{2}-2 l^{2}}{2\left(L^{2}+l^{2}\right)}$, which is precisely the value of $r_{\mathrm{H}}$ that is $\leq 1$. If $\frac{l}{L}<\frac{1}{\sqrt{2}}$, then $p_{n, 2}(t) \geq 0$ for all $r \in[-1,0]$. If $\frac{l}{L}>\frac{1}{\sqrt{2}}$, then $p_{n, 2}(t) \geq 0$ for all $r \in\left[-1, r_{\mathrm{H}}\right]$. We infer that $r \in\left[-1, \min \left(r_{\mathrm{H}}, 0\right)\right]$. The admissible domain is depicted in Fig. 5.

Remark 7 The sticking assumption introduces a jump in $\dot{x}$, and hence in $\dot{q}_{\text {tan }}$ (indeed from the kinematic constraints one has $\dot{x}\left(t^{+}\right)=-r \dot{x}\left(t^{-}\right)$and $\left.\dot{q}_{\tan }=\sqrt{m} \dot{x}\right)$ and modifies the impulse form. Hence, it corresponds to some general restitution matrix $\mathcal{E}$ as in (14) with discontinuous $\dot{q}_{\tan }$. Taking into account that we may redo the same analysis when the block rotates around $B$ and hits the ground at $A$ to describe the rocking over a complete cycle, we obtain $\sqrt{m} \dot{x}\left(t^{+}\right)=-\frac{l}{2} \sqrt{m} \dot{\theta}\left(t^{+}\right)=-r \sqrt{m} \dot{x}\left(t^{-}\right)$so that $\dot{q}_{\mathrm{tan}}\left(t^{+}\right)=-r \dot{q}_{\mathrm{tan}}\left(t^{-}\right)$, and 
Fig. 5 The admissible domain for $r$ (sticking rocking motion)

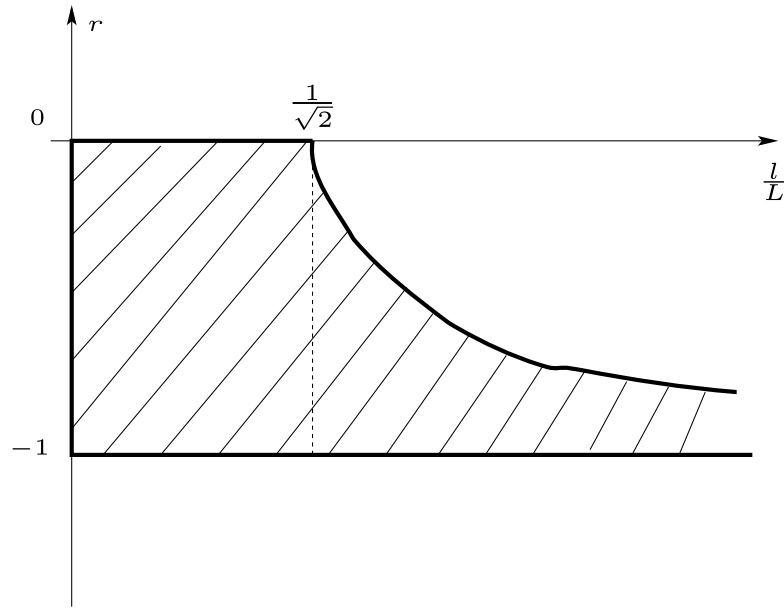

$\mathcal{E}=\left(\begin{array}{ccc}0 & -r & 0 \\ -r & 0 & 0\end{array}\right)$, with $\mathcal{E}^{T} \mathcal{E}=\operatorname{diag}\left(r^{2}\right)$. In the case of the restitution law of Sect. 3.4 one obtains $\mathcal{E}=\left(\begin{array}{ccc}0 & e_{n, 21} & 0 \\ e_{n, 21} & 0 & 0 \\ 0 & 0 & -e_{n, 21}\end{array}\right)$ if one imposes the same coefficient for the two subcycles of the rocking motion, i.e., $e_{n, 12}=e_{n, 21}$.

Let us now study the angular velocity restitution for sticking free-rocking, and assuming that $\lambda_{n, 2}$ is a function (there is no Dirac measure acting at $A$ when the block collides at $B$ ). Imposing $p_{n, 2}(t)=0$ in (45) yields $r=\frac{L^{2}-2 l^{2}}{2\left(L^{2}+l^{2}\right)}=r_{\mathrm{H}}$. Since rocking implies that $\dot{\theta}\left(t^{+}\right)$and $\dot{\theta}\left(t^{-}\right)$have the same sign, necessarily $r_{\mathrm{H}} \leq 0$, equivalently $\frac{l}{L}>\frac{1}{\sqrt{2}}$.

\subsubsection{Half-rocking block}

The half-rocking implies $r>0$ (the angular velocity reverses its sign after the impact at $B)$, while $\dot{q}_{\text {norm }, 2}\left(t^{+}\right)=\dot{q}_{\text {norm }, 2}\left(t^{-}\right)=0$, i.e., $\dot{y}\left(t^{+}\right)=\frac{L}{2} \dot{\theta}\left(t^{+}\right)$and $\dot{y}\left(t^{-}\right)=\frac{L}{2} \dot{\theta}\left(t^{-}\right)$. Also $\dot{\theta}\left(t^{-}\right)<0$ and $\dot{\theta}\left(t^{+}\right)>0$. The impact dynamics are thus rewritten as

$$
\left\{\begin{array}{l}
\frac{m L}{2}\left(\dot{\theta}\left(t^{+}\right)-\dot{\theta}\left(t^{-}\right)\right)=p_{n, 1}(t)+p_{n, 2}(t) \\
\frac{2 I_{G}}{L}\left(\dot{\theta}\left(t^{+}\right)-\dot{\theta}\left(t^{-}\right)\right)=p_{n, 1}(t)-p_{n, 2}(t) .
\end{array}\right.
$$

It follows that $p_{n, 1}(t)=-(r+1)\left(\frac{m L}{2}+\frac{2 I_{G}}{L}\right) \dot{\theta}\left(t^{-}\right)$that is always nonnegative since the energetic constraint implies $r \in[-1,1]$, while $p_{n, 2}(t)=(r+1)\left(-\frac{m L}{2}+\frac{2 I_{G}}{L}\right) \dot{\theta}\left(t^{-}\right)$. Therefore, $p_{n, 2}(t) \geq 0$ if and only if $-\frac{m L}{2}+\frac{2 I_{G}}{L} \geq 0$ and $r \leq-1$, or $-\frac{m L}{2}+\frac{2 I_{G}}{L} \leq 0$ and $r \geq-1$. Thus, this is equivalent to $l \leq \sqrt{2} L$ and $r \in(0,1]$.

Finally assuming that $p_{n, 2}(t)=0$, one finds that $\frac{m L}{2}\left(\dot{\theta}\left(t^{+}\right)-\dot{\theta}\left(t^{-}\right)\right)=p_{n, 1}(t)=$ $\frac{2 I_{G}}{L}\left(\dot{\theta}\left(t^{+}\right)-\dot{\theta}\left(t^{-}\right)\right)$so that $\frac{m L}{2}=\frac{2 I_{G}}{L}$ which is equivalent to $l=\sqrt{2} L$, i.e., $\theta_{12}=\frac{\pi}{2}$. Halfrocking implies $r>0$ which together with the energetic constraint implies that $r \in[0,1]$.

Remark 8 The above may also be considered as a generalization of the analysis made in [30]. In particular, Figs. 3 and 5 are similar to Fig. 10 in [30]. 


\section{Recapitulation and comments}

Several kinetic angles appeared in the foregoing calculations that correspond at $\theta=0$ to (a) $l=\frac{1}{\sqrt{2}} L$, (b) $l=\sqrt{2} L$, (c) $\frac{l}{L}=\frac{-3 \mu+\sqrt{9 \mu^{2}+8}}{2}$, (d) $\frac{l}{L}=\frac{3 \mu+\sqrt{9 \mu^{2}+8}}{2}$. Notice that when $\mu=0$ the aspect ratios in cases (c) and (d) are equal to $\sqrt{2}$. They are given by $\theta_{12}=\pi-$ $\arccos \frac{l^{2}-2 L^{2}}{4 L^{2}+l^{2}}$; see Sect. 2.2 for the general formula. The values in (c) and (d) are obtained from $e_{c, 1}^{+}=0$ and $e_{c, 1}^{-}=0$ in Sect. 3.6.3. One therefore gets (a) $\gamma \triangleq \pi-\arccos \left(-\frac{1}{3}\right)<\frac{\pi}{2}$, (b) $\beta=\frac{\pi}{2}$, (c) $\delta=\pi-\arccos \left(\frac{18 \mu^{2}-6 \mu \sqrt{9 \mu^{2}+8}}{18 \mu^{2}-6 \mu \sqrt{9 \mu^{2}+8}+24}\right)$, (d) $\zeta=\pi-\arccos \left(\frac{18 \mu^{2}+6 \mu \sqrt{9 \mu^{2}+8}}{18 \mu^{2}+6 \mu \sqrt{9 \mu^{2}+8}+24}\right)$, respectively. The value $\theta_{12}=\frac{\pi}{3}$ corresponds to the limit case $l=0$. We have examined the capabilities of a generalized restitution law, under the energetic, kinematic, and kinetic constraints. In the frictionless case, this boils down to finding a restitution matrix $\mathcal{E}_{n}$ such that, given preimpact velocities one has

$$
\left\{\begin{array}{l}
\mathcal{E}_{n}^{T} A(q) \mathcal{E}_{n} \leq A(q), \text { or }\left|\lambda_{\max }\left(\mathcal{E}_{n}\right)\right| \leq 1 \text { if } \mathcal{E}_{n}=\mathcal{E}_{n}^{T} \\
\left(\mathbf{n}_{q}(t)^{T} \nabla f(q(t))\right)^{-1}\left(\mathcal{E}_{n}+I_{n}\right) \dot{q}_{\text {norm }}\left(t^{-}\right) \leq 0 \\
\mathcal{E}_{n} \dot{q}_{\text {norm }}\left(t^{-}\right) \leq 0 \\
C \dot{q}_{\text {norm }}\left(t^{-}\right)=0
\end{array}\right.
$$

for some matrix $C$ with entries 0 or 1 . It is clear that the energetic consistency can be stated for a given preimpact velocity as a sufficient condition. However, (13) states a necessary and sufficient condition for a given $\dot{q}_{\text {norm }}\left(t^{-}\right)$and may involve different coefficients of $\mathcal{E}_{n}$ depending on $\dot{q}_{\text {norm }}\left(t^{-}\right)$. Therefore, by varying the preimpact velocity different constraints may be found on the restitution coefficients (see Sect. 7 for an example). This is a particular feature of multiple impacts compared to single ones. The results are recapitulated in the Table 1 . We have done all the calculations when the block rotates around $A$ and collides the ground at $B$. By doing the reverse (impact at $A$ and rotation around $B$ ), one infers similar values for the restitution coefficients $e_{n, 2}$ and $e_{n, 12}$, which we do not indicate in the table to lighten the presentation. $\theta_{12, c}$ denotes the critical kinetic angles that separate the admissible domains for $e_{n, 21}$ or $r$ into two subdomains: one for which the coefficient can be chosen inside $[0,1]$, one in which the coefficient has to be taken in a smaller interval (see Figs. 2, 3 , and 4).

- The fact that $e_{n, 21}=0$ for half-rocking means that a diagonal $\mathcal{E}_{n}$ is sufficient in this case.

- In a companion paper, another impact law is used that allows one to model slip and stick behaviors outside impacts and during impacts; see [56] and [57]. The results reported therein prove that indeed the basic assumptions for the Housner approach (perfectly sticking contact points) hold only for high enough friction and $\frac{l}{L} \gg \sqrt{2}$, i.e., $\theta_{12} \in\left(\frac{\pi}{2}, \pi\right)$. Otherwise, and in particular for realistic values of the friction, such assumptions are never met and the rocking motion with all-sticking contact/impact points occurs only if the blocks are slender enough,

- $r_{\mathrm{H}}$ is an upperbound for the admissible $r$ for sticking free-rocking,

- $-r$ is an off-diagonal entry of $\mathcal{E}_{n}$, hence can be interpreted as a coefficient that takes into account "distance effects" between the two contact points,

- rocking motion cannot be modeled without the introduction of $r$ or equivalently $e_{n, 21}$,

- the classical angular restitution coefficient is equivalent to a generalized restitution law with nondiagonal restitution matrix $\mathcal{E}_{n}$, 


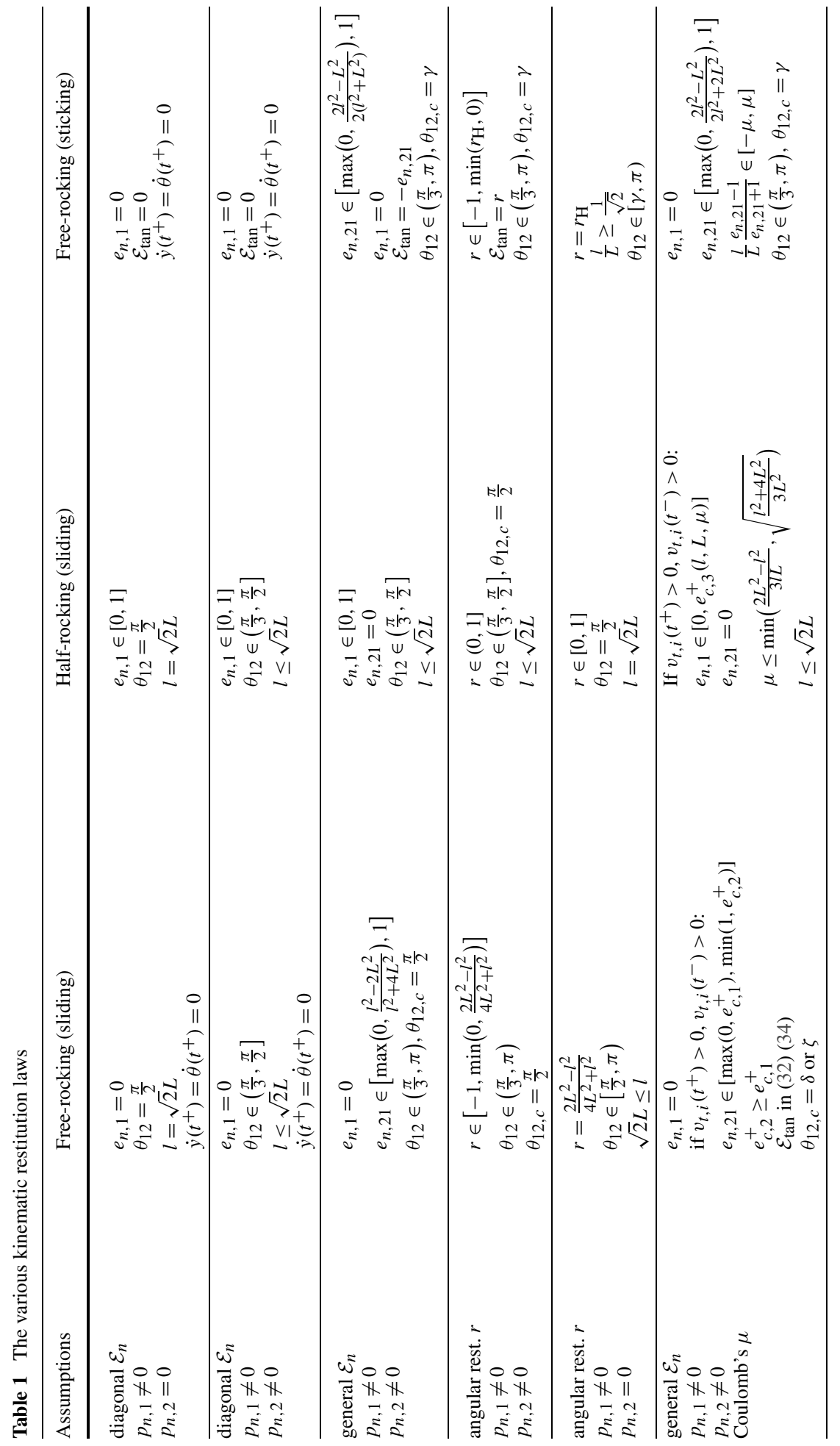


- for free-rocking without Coulomb's friction, there is a critical aspect ratio equal to $\sqrt{2}$ in the sliding case $\left(\theta_{12, c}=\frac{\pi}{2}\right)$, and to $\frac{1}{\sqrt{2}}$ for the sticking case $\left(\theta_{12, c}=\gamma\right)$. Below (resp. above) $\theta_{12, c}$ the choice for $e_{n, 21}$ (resp. for $r$ ) is dictated only by the energetical and kinematic constraints. Otherwise, they have to be chosen in a smaller interval. The critical aspect ratio for the sticking case corresponds to $r_{\mathrm{H}}=0$,

- the kinematic laws are able to describe half-rocking only for flat blocks (see the second column of Table 1),

- let us denote $r_{\text {sli }}=\frac{2 L^{2}-l^{2}}{4 L^{2}+l^{2}}$ the value of $r$ that is obtained from the moment of momentum conservation $\sigma_{B}\left(t^{+}\right)=\sigma_{B}\left(t^{-}\right)$in the sliding case (see Sect. 3.7.2). Then $r_{\text {sli }}>r_{\mathrm{H}}$ for all aspect ratios except for the limit values $\frac{l}{L}=0$ and $\frac{l}{L}=+\infty$ (denoting $\alpha=\frac{l^{2}}{L^{2}}$ it follows that $r_{\text {sli }}>r_{\mathrm{H}}$ is equivalent to $\alpha>0$ ). This means that assuming sticking constrains more the choice of the restitution coefficient than assuming sliding. $r_{\text {sli }}$ and $r_{\mathrm{H}}$ can thus be considered as two critical values for $r$.

- From (10), it follows that $\theta_{12}=\arccos \left(r_{\mathrm{sli}}\right)$. Let us denote $\theta_{\mathrm{H}}=\arccos \left(r_{\mathrm{H}}\right)$. We may thus write that $r \in\left[-1, \min \left(0, \cos \left(\theta_{12}\right)\right)\right]$ for the sliding rocking while $r \in$ $\left[-1, \min \left(0, \cos \left(\theta_{\mathrm{H}}\right)\right)\right]$ for sticking rocking. One has $\theta_{\mathrm{H}}>\theta_{12}$. This means that the stick assumption overestimates the kinetic angle of the block/ground system, or equivalently underestimates the aspect ratio $\frac{l}{L}$ : one thinks that the block is slender enough but is not. In proportion as the block becomes more slender, $\theta_{\mathrm{H}}$ approaches $\theta_{12}$. We recover the fact that the sticking assumption works better for very slender blocks, as confirmed experimentally in [30] and experimentally in [56, 57]. For less slender blocks, there usually exist rebound phases at the impacting corner, and stick/slip modes both during and outside the impacts [57].

- In all the frictionless sliding cases, one has $\mathcal{E}_{\tan }=-1$ since $\dot{x}$ is continuous at the impacts.

\section{General $\mathcal{E}_{n}, p_{n, 1}(t) \neq 0, p_{n, 2}(t) \neq 0$, rebound at $B$}

In the foregoing sections, we have analyzed the capabilities of the generalized impact law (12) (14) and compared it to the angular restitution law, for perfect rocking without tangential effects or with perfect contact sticking. In particular, it has been imposed always $e_{n, 1}=0$. Let us now study the case when the block rotates around $A$ and sticks in the tangential direction, hits the ground at $B$, and we allow for $e_{n, 1}>0$, i.e., a positive restitution coefficient at $B$. This may be motivated by the fact that in most of the reported experiments, rocking occurs while $e_{n, 1} \neq 0[30,45]$. As will be shown the uniqueness of the restitution parameters (coefficients) may be lost for a given energetic behavior. The kinematic constraints at $A$ are $\dot{x}\left(t^{-}\right)=-\frac{l}{2} \dot{\theta}\left(t^{-}\right), \dot{q}_{\text {norm, } 2}\left(t^{-}\right)=\dot{y}\left(t^{-}\right)-\frac{L}{2} \dot{\theta}\left(t^{-}\right)=0$. The restitution law is $\dot{q}_{\text {norm }}\left(t^{+}\right)=-\mathcal{E}_{n} \dot{q}_{\text {norm }}\left(t^{-}\right)$, which gives $\dot{q}_{\text {norm }, 1}\left(t^{+}\right)=-e_{n, 1} \dot{q}_{\text {norm }, 1}\left(t^{-}\right)-e_{n, 12} \dot{q}_{\text {norm }, 2}\left(t^{-}\right)=$ $-e_{n, 1} \dot{q}_{\text {norm }, 1}\left(t^{-}\right)$, and $\dot{q}_{\text {norm }, 2}\left(t^{+}\right)=-e_{n, 21} \dot{q}_{\text {norm }, 1}\left(t^{-}\right)-e_{n, 2} \dot{q}_{\text {norm }, 2}\left(t^{-}\right)=-e_{n, 21} \dot{q}_{\text {norm }, 1}\left(t^{-}\right)$. The impact dynamics are given by

$$
\left\{\begin{array}{l}
m\left(\dot{x}\left(t^{+}\right)-\dot{x}\left(t^{-}\right)\right)=p_{t, 1}+p_{t, 2} \\
m\left(\dot{y}\left(t^{+}\right)-\dot{y}\left(t^{-}\right)\right)=p_{n, 1}+p_{n, 2} \\
I_{G}\left(\dot{\theta}\left(t^{+}\right)-\dot{\theta}\left(t^{-}\right)\right)=\frac{L}{2}\left(p_{n, 1}-p_{n, 2}\right)+\frac{l}{2}\left(p_{t, 1}+p_{t, 2}\right) .
\end{array}\right.
$$

For the moment, we have 6 unknowns and only 5 equations. A sixth equation is added by defining $\mathcal{E}_{\tan }=e_{t, 3}$, i.e., $\dot{x}\left(t^{+}\right)=-e_{t, 3} \dot{x}\left(t^{-}\right) \Leftrightarrow \dot{q}_{\tan }\left(t^{+}\right)=-e_{t, 3} \dot{q}_{\tan }\left(t^{-}\right)$since 
$; \dot{q}_{\tan }=\sqrt{m} \dot{x}$. Combining the kinematic constraints and the impact dynamics together with the restitution law, one obtains the following:

$$
\left\{\begin{array}{l}
\dot{x}\left(t^{+}\right)=-e_{t, 3} \dot{x}\left(t^{-}\right) \\
\dot{y}\left(t^{+}\right)=-\frac{1}{2}\left(e_{n, 1}+e_{n, 21}\right) L \dot{\theta}\left(t^{-}\right) \\
\dot{\theta}\left(t^{+}\right)=\left(-e_{n, 1}+e_{n, 21}\right) \dot{\theta}\left(t^{-}\right) .
\end{array}\right.
$$

After some calculations, the kinetic energy loss is given by

$$
\begin{aligned}
T_{L}(t) & =\frac{m}{8}\left[l^{2}\left(e_{t, 3}^{2}-1\right)+L^{2}\left(e_{n, 1}+e_{n, 21}\right)^{2}-L^{2}+\frac{l^{2}+L^{2}}{3}\left(\left(-e_{n, 1}+e_{n, 21}\right)^{2}-1\right)\right] \dot{\theta}^{2}\left(t^{-}\right) \\
& =\frac{m}{8} \dot{\theta}^{2}\left(t^{-}\right)\left[-\frac{4 l^{2}+4 L^{2}}{3}+l^{2} e_{t, 3}^{2}+L^{2}\left(e_{n, 1}+e_{n, 21}\right)^{2}+\frac{l^{2}+L^{2}}{3}\left(-e_{n, 1}+e_{n, 21}\right)^{2}\right],
\end{aligned}
$$

from which it follows that $T_{L} \leq 0$ is equivalent to:

$$
\left(l^{2}+4 L^{2}\right)\left(e_{n, 1}^{2}+e_{n, 21}^{2}\right)+\left(4 L^{2}-2 l^{2}\right) e_{n, 1} e_{n, 21}+3 l^{2} e_{t, 3}^{2} \leq 4\left(l^{2}+L^{2}\right) .
$$

Defining $e_{1} \triangleq e_{n, 1}+e_{n, 21}, e_{2} \triangleq e_{n, 21}-e_{n, 1}, e_{32} \triangleq e_{t, 3}$, one finds equivalently:

$$
L^{2} e_{1}^{2}+\frac{l^{2}+L^{2}}{3} e_{2}^{2}+l^{2} e_{3}^{2} \leq \frac{4}{3}\left(l^{2}+L^{2}\right) .
$$

Clearly, for a given energetic behavior (for instance, the lossless case $T_{L}=0$ ) the coefficients live on an ellipsoid $\mathcal{S}_{e}$, and are nonunique. This was already known for chains of balls [8] but is shown here for the block for the first time. One has $p_{n, 1}(t)+p_{n, 2}(t)=-\frac{m L}{2}\left(e_{n, 1}+\right.$ $\left.e_{n, 21}+1\right) \dot{\theta}\left(t^{-}\right)$, and $p_{n, 1}(t)-p_{n, 2}(t)=\left[\frac{2 I_{G}}{L}\left(e_{n, 1}-e_{n, 21}+1\right)-\frac{m l^{2}}{2 L}\left(e_{t, 3}+1\right)\right] \dot{\theta}\left(t^{-}\right)$. Hence, the kinetic constraints are given by

$$
p_{n, 1}(t) \geq 0 \quad \Leftrightarrow \quad\left(l^{2}+4 L^{2}\right) e_{n, 1}+\left(2 L^{2}-l^{2}\right) e_{n, 21}+3 l^{2} e_{t, 3}+7 l^{2}+4 L^{2} \geq 0
$$

and

$$
p_{n, 2}(t) \geq 0 \quad \Leftrightarrow \quad\left(2 L^{2}-l^{2}\right)\left(e_{n, 1}+e_{n, 21}\right)-3 l^{2} e_{t, 3}+2\left(L^{2}-2 l^{2}\right) \geq 0 .
$$

These two constraints define two subspaces $\mathcal{S}_{1}$ and $\mathcal{S}_{2}$ of the thee-dimensional space of the restitution coefficients, and the admissible coefficients must lie in $\mathcal{S}_{e} \cap \mathcal{S}_{1} \cap \mathcal{S}_{2}$.

\section{Tangential (local) restitution}

At an impact time, the tangential velocities of $A$ and $B$ are equal to $v_{t, i}=\dot{x}+\frac{l}{2} \dot{\theta}$ for $i=$ 1,2 . Using the restitution law in (12) and the kinematic constraints of rocking, one finds $v_{t, i}\left(t^{+}\right)=-e_{t, 3} \dot{x}\left(t^{-}\right)+\frac{l}{2}\left(-e_{n, 1}+e_{n, 21}\right) \dot{\theta}\left(t^{-}\right)$. Starting from $v_{t, i}\left(t^{+}\right)=e_{t} v_{t, i}\left(t^{-}\right)$for some tangential restitution coefficient, one obtains $v_{t, i}\left(t^{+}\right)=e_{t} \dot{x}\left(t^{-}\right)+\frac{l}{2} e_{t} \dot{\theta}\left(t^{-}\right)$. The relation between the generalized coefficients and the local tangential coefficient is therefore

$$
e_{t}=-e_{t, 3}=-e_{n, 1}+e_{n, 21} \text {. }
$$


We infer that imposing a tangential restitution at the local tangential velocities of the contact points, introduces a constraint on the generalized coefficients $e_{n, 1}, e_{n, 2}, e_{n, 21}$. The fact that there exists constraints between various kinematic restitution coefficients is known in the case of single impacts [6,8]. Let us impose further sticking at the end of the impact at $B: v_{t, 1}\left(t^{+}\right)=\dot{x}\left(t^{+}\right)+\frac{l}{2} \dot{\theta}\left(t^{+}\right)=0$, so that $\dot{x}\left(t^{+}\right)=-\frac{l}{2} \dot{\theta}\left(t^{+}\right)$. This imposes that $e_{t}=0$ so that $e_{t, 3}=0$ and $e_{n, 1}=e_{n, 21}$. Thus the restitution matrix takes the form $\mathcal{E}=\left(\begin{array}{ccc}e_{n} & e_{n} & 0 \\ e_{n} & e_{n} & 0 \\ 0 & 0 & 0\end{array}\right)$. If perfect rocking is modeled then $e_{n, 1}=0$, and consequently $\mathcal{E}_{n}=0$. If we now impose $e_{n, 1}=0$, then $e_{t, 3}=-e_{n, 21}$ and $\mathcal{E}=\left(\begin{array}{ccc}0 & e_{n, 21} & 0 \\ e_{n, 21} & 0 & 0 \\ 0 & 0 & -e_{n, 21}\end{array}\right)$. The results in Remark 7 are recovered. It is interesting to note that in this last case, the local and the generalized tangential restitutions are identical. Also, the off-diagonal entries of $\mathcal{E}_{n}$ and the local tangential coefficients are not independent coefficients, as shown in (55).

The angular restitution coefficient $r$ is a tangential restitution coefficient when interpreted within the generalized impact law (14). Thus, it is not associated with a unilateral constraint and has no reason to be a signed coefficient.

Remark 9 The physical phenomenon that is responsible for sticking of the contact points is Coulomb's friction. Thus the sticking free-rocking motion should not be postulated a priori, but should be the result of the effects of Coulomb's friction. The coefficients $e_{n, 21}, e_{n, 3}$, or $e_{t}$ may be seen as macroscopic parameters that more or less accurately model tangential effects like friction and possible tangential compliance (remind that contrarily to the normal compliance, the tangential compliance plays no role if there is no friction that triggers some tangential deformation). They can be considered on a similar footing than the impulse ratio of Brach [6] for single impacts. It is clear that the local tangential restitution and the generalized one applied on $\dot{q}_{\text {tan }}$ are not the same in general. The local tangential restitution coefficient introduced in [40, Sect. 6] (see Eq. (6.4) in that paper) is a local tangential restitution equal to $e_{t}$. This shows that the generalized kinematic law with $e_{n, 1}=0, e_{n, 21}$, and $e_{t, 3}$ can be recast in the numerical framework proposed in [40]. There has been a significant literature about tangential restitution for the single impact between two rough bodies, and its link with Coulomb's friction (see, e.g., [8, Chap. 4], [35, 50, 51, 53]). Redoing a similar analysis as in [51] to better understand the 2-impact phenomenon would certainly be useful.

\section{Application to a simple granular system: four aligned balls}

Let us consider the system made of four aligned balls with mass $m$ and radius $R$, sliding without friction on a ground (a 4-ball Newton's cradle), as shown in Fig. 6. In the granular matter literature, this is known as a monodisperse chain, in the conservative case. The generalized coordinate vector is $q=\left(q_{1} q_{2} q_{3} q_{4}\right)^{T}, q_{i}$ being the position of the gravity center of each ball. The mass matrix is $M=\operatorname{diag}(m)$. The three unilateral constraints are given by $f_{1}(q)=q_{2}-q_{1}-2 R \geq 0, f_{2}(q)=q_{3}-q_{2}-2 R \geq 0, f_{3}(q)=q_{4}-q_{3}-2 R \geq 0$. Therefore, $\nabla f_{1}(q)=\left(\begin{array}{llll}-1 & 1 & 0 & 0\end{array}\right)^{T}, \nabla f_{2}(q)=\left(\begin{array}{llll}0 & -1 & 1 & 0\end{array}\right)^{T}, \nabla f_{3}(q)=\left(\begin{array}{lllll}0 & 0 & -1 & 1\end{array}\right)^{T}$. After some calculations, one finds $\dot{q}_{\text {norm }, 1}=\frac{1}{\sqrt{2 m}}\left(-\dot{q}_{1}+\dot{q}_{2}\right), \dot{q}_{\text {norm }, 1}=\frac{1}{\sqrt{2 m}}\left(-\dot{q}_{2}+\dot{q}_{3}\right)$, $\dot{q}_{\text {norm }, 3}=\frac{1}{\sqrt{2 m}}\left(-\dot{q}_{3}+\dot{q}_{4}\right)$, and $\dot{q}_{\text {tan }}=\frac{\sqrt{m}}{2}\left(\dot{q}_{1}+\dot{q}_{2}+\dot{q}_{3}+\dot{q}_{4}\right)$. Since there is no tangential effect in such a system, we may set $\mathcal{E}_{\tan }=-1$. 
Fig. 6 Four aligned balls

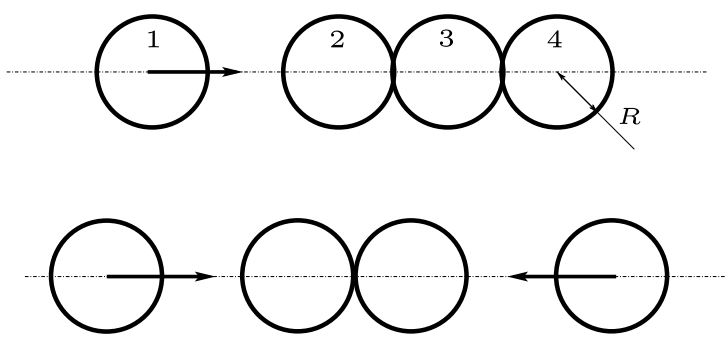

\subsection{A first preimpact velocity}

Suppose that $\dot{q}_{1}\left(t^{-}\right)=1 \mathrm{~m} / \mathrm{s}$, while $\dot{q}_{i}\left(t^{-}\right)=0, i=2,3,4$, and the balls $2,3,4$ are in contact at the impact time when the first ball collides into them; see Fig. 6(a). This is a 3-impact according to Definition 1. From (12), we obtain

$$
\dot{q}_{\text {norm }}\left(t^{+}\right)=\mathcal{E}_{n}\left(\begin{array}{c}
\frac{1}{\sqrt{2 m}} \\
0 \\
0
\end{array}\right),
$$

that is,

$$
\left\{\begin{array}{l}
\dot{q}_{\text {norm }, 1}\left(t^{+}\right)=\frac{1}{\sqrt{2 m}} e_{n, 1} \\
\dot{q}_{\text {norm }, 1}\left(t^{+}\right)=\frac{1}{\sqrt{2 m}} e_{n, 21} \\
\dot{q}_{\text {norm }, 3}\left(t^{+}\right)=\frac{1}{\sqrt{2 m}} e_{n, 31} .
\end{array}\right.
$$

Clearly, the off-diagonal restitution coefficients $e_{n, 21}$ and $e_{n, 31}$ allow us to span the whole space of admissible post-impact velocities $\dot{q}_{\text {norm }}\left(t^{+}\right) \geq 0$. It readily follows from (57) that the kinematic constraints imply that $e_{n, 1} \geq 0, e_{n, 21} \geq 0, e_{n, 31} \geq 0$. The impact dynamics are given by

$$
\left\{\begin{array}{l}
m\left(\dot{q}_{1}\left(t^{+}\right)-1\right)=-p_{12}(t) \\
m \dot{q}_{2}\left(t^{+}\right)=p_{12}(t)-p_{23}(t) \\
m \dot{q}_{3}\left(t^{+}\right)=p_{23}(t)-p_{34}(t) \\
m \dot{q}_{4}\left(t^{+}\right)=p_{34}(t) .
\end{array}\right.
$$

The set of equations in (57) and (58) provides 7 equations for 7 unknowns. This is equivalent to use (57) and (11). One finds

$$
\left\{\begin{array}{l}
\dot{q}_{1}\left(t^{+}\right)=\frac{1-3 e_{n, 1}-2 e_{n, 21}-e_{n, 31}}{4} \\
\dot{q}_{2}\left(t^{+}\right)=\frac{1+e_{n, 1}-2 e_{n, 21}-e_{n, 31}}{4} \\
\dot{q}_{3}\left(t^{+}\right)=\frac{1+e_{n, 1}+2 e_{n, 21}-e_{n, 31}}{4} \\
\dot{q}_{4}\left(t^{+}\right)=\frac{1+e_{n, 1}+2 e_{n, 21}+3 e_{n, 31}}{4} \\
p_{12}(t)=m \frac{3+3 e_{n, 1}+2 e_{n, 21}+e_{n, 31}}{4} \\
p_{23}(t)=m \frac{1+e_{n, 1}+2 e_{n, 21}+e_{n, 31}}{2} \\
p_{34}(t)=m \frac{1+e_{n, 1}+2 e_{n, 21}+3 e_{n, 31}}{4} .
\end{array}\right.
$$


We deduce the kinematic constraints for the three coefficients imply that the kinetic constraints $p_{12}(t) \geq 0, p_{23}(t) \geq 0, p_{34}(t) \geq 0$, are satisfied. Using (13) for the particular $\dot{q}_{\text {norm }}\left(t^{-}\right)$, one finds that the energetical consistency holds if and only if

$$
e_{n, 1}^{2}+e_{n, 21}^{2}+e_{n, 31}^{2} \leq 1
$$

One infers that the three restitution coefficients have to be nonnegative and in the ellipsoid defined by (60). For instance in the lossless (conservative) case, one finds that the coefficients should lie on the ellipse $e_{n, 1}^{2}+e_{n, 21}^{2}+e_{n, 31}^{2}=1$. Contrarily to single impacts, the energetical behavior does not define a unique set of restitution coefficients. This means that given the data: preimpact velocity, restitution matrix, kinematic, and kinetic consistencies, and the energetical behavior, one is not able to compute a unique post-impact velocity. This is due to the fact that a fundamental mechanical information is lacking in the model, related to the vibrational (waves) effects through the chain: the flexibilities at the contact points.

Remark 10 The above analysis can be easily extended to $n$-ball chains. There is no tangential effect in such chains of balls, which implies that $\dot{q}_{\text {tan }}$ is always a scalar and $\dot{q}_{\tan }\left(t^{+}\right)=\dot{q}_{\tan }\left(t^{-}\right)$merely translates the conservation of the linear momentum. Therefore, contrarily to the rocking block where some motions can be described only if $\mathcal{E}_{\tan }$ is different from $-I_{n-m}$ (see Remark 7), for aligned chains of balls one always has $\mathcal{E}_{\tan }=-1$. Incidentally, when applying Moreau's impact law that is $\mathcal{E}_{n}=\operatorname{diag}(e)$, one finds that $\dot{q}_{\text {norm, } 1}\left(t^{+}\right) \geq 0$, $\dot{q}_{\text {norm }, 1}\left(t^{+}\right)=\dot{q}_{\text {norm }, 3}\left(t^{+}\right)=0$ and $e \in[0,1]$, which is consistent with Proposition 1 . The postimpact velocity is on the so-called Moreau's half-line of the admissible set of post-impact velocities [23].

Remark 11 As shown in [23, Sects. 3.8, 5.6] Moreau's rule is not able to describe the whole set of admissible post-impact velocities, but only a very small subset of it. The analysis in this section shows that the proposed generalized impact rule allows to span a much broader set of post-impact velocities.

\subsection{A second preimpact velocity}

Let us now choose another $\dot{q}_{\text {norm }}\left(t^{-}\right)$with $\dot{q}_{1}\left(t^{-}\right)=1 \mathrm{~m} / \mathrm{s}, \dot{q}_{2}\left(t^{-}\right)=\dot{q}_{3}\left(t^{-}\right)=0, \dot{q}_{4}\left(t^{-}\right)=$ $-2 \mathrm{~m} / \mathrm{s}$; see Fig. 6(b). Thus, $\dot{q}_{\text {norm }, 1}\left(t^{-}\right)=\frac{-1}{\sqrt{2 m}}, \dot{q}_{\text {norm }, 3}\left(t^{-}\right)=\frac{-2}{\sqrt{2 m}}$, and $\dot{q}_{\text {norm }, 1}\left(t^{-}\right)=0$. The energetical consistency yields

$$
e_{n, 1}^{2}+4 e_{n, 13}^{2}+e_{n, 21}^{2}+4 e_{n, 23}^{2}+e_{n, 31}^{2}+4 e_{n, 3}^{2}+4 e_{n, 1} e_{n, 13}+4 e_{n, 21} e_{n, 23}+4 e_{n, 31} e_{n, 3} \leq 5
$$

The kinematic consistency yields

$$
\left\{\begin{array}{l}
e_{n, 1}+2 e_{n, 13} \geq 0 \\
e_{n, 21}+2 e_{n, 23} \geq 0 \\
e_{n, 31}+2 e_{n, 3} \geq 0
\end{array}\right.
$$


The percussion vector is equal to

$$
\left\{\begin{array}{l}
\dot{q}_{1}\left(t^{+}\right)=\frac{-1-3 e_{n, 1}-6 e_{n, 13}-2 e_{n, 21}-4 e_{n, 23}-e_{n, 31}-2 e_{n, 3}}{4} \\
\dot{q}_{2}\left(t^{+}\right)=\frac{-1+e_{n, 1}+2 e_{n, 13}-2 e_{n, 21}-4 e_{n, 23}-e_{n, 31}-2 e_{n, 3}}{4} \\
\dot{q}_{3}\left(t^{+}\right)=\frac{-1+e_{n, 1}+2 e_{n, 13}+2 e_{n, 21}+4 e_{n, 23}-e_{n, 31}-2 e_{n, 3}}{4} \\
\dot{q}_{4}\left(t^{+}\right)=\frac{-1+e_{n, 1}+2 e_{n, 13}+2 e_{n, 21}+4 e_{n, 23}+3 e_{n, 31}+6 e_{n, 3}}{4} \\
p_{12}(t)=m \frac{5+3 e_{n, 1}+6 e_{n, 13}+2 e_{n, 21}+4 e_{n, 23}+e_{n, 31}+2 e_{n, 3}}{4} \\
p_{23}(t)=m \frac{3+2 e_{n, 1}+2 e_{n, 13}+2 e_{n, 21}+4 e_{n, 23}+2 e_{n, 31}+2 e_{n, 3}}{2} \\
p_{34}(t)=m \frac{7+e_{n, 1}+2 e_{n, 13}+2 e_{n, 21}+4 e_{n, 23}+3 e_{n, 31}+6 e_{n, 3}}{4} .
\end{array}\right.
$$

The kinetic consistency conditions follow from $p_{12}(t) \geq 0, p_{23}(t) \geq 0, p_{34}(t) \geq 0$. It is clear from (62) and (63) that nonnegative restitution coefficients which also satisfy (61) are a subset of kinematically, kinetically, and energetically consistent coefficients. Consider the conservative case (with equality in (61)). It is then possible that some coefficients be larger than 1 while being admissible.

In all Sects. 3.4, 5, 7.1, and 7.2, the preimpact velocity is such that not all the entries of $\mathcal{E}_{n}$ play a role in the analysis of the multiple impact, but only some of them. If one applies the general criterion of Proposition 1, then other, different upperbounds for the restitution coefficients will be found. For instance for the rocking block where $\mathcal{E}_{n}=\left(\begin{array}{cc}e_{n, 1} & e_{n, 12} \\ e_{n, 21} & e_{n, 2}\end{array}\right)$, one finds applying Proposition 1:

$$
e_{n, 1}+e_{n, 2}+\sqrt{\left(e_{n, 1}-e_{n, 2}\right)^{2}+4 e_{n, 12}^{2}} \leq 2
$$

Choosing $e_{n, 1}=0$ as in Sect. 3.4 obviously yields different upperbound for $e_{n, 21}=e_{n, 12}$ than in Fig. 3, except if one chooses also $e_{n, 2}=0$.

\subsection{Discussion}

Several questions arise from the rocking block and the chain of balls examples:

(i) Several upperbounds for the restitution coefficients may be derived, depending on the pre-impact velocities and/or on the system's constraints. On the other hand, general upperbounds guaranteeing energetical consistency for any type of constraints and initial velocities may also be derived. In general, the energetical behavior is not sufficient to assure the coefficients uniqueness. This may be due, in part, to the fact that kinematic laws are unable to correctly handle wave effects, which are primarily due to flexibilities, and are responsible for energy dispersion. Which ones are the correct parameters, however?

(ii) The proposed impact law is an extension of Moreau's laws [1, 8, 22, 23, 40] as it is expressed with generalized velocities. In the case of the ground/block system both laws may even be identical, see Remark 9, which shows that the generalized law can be implemented in a time-stepping code based on complementarity tools. Is there a way to generalize this conclusion?

(iii) The mechanical meaning of those generalized restitution coefficients is not always clear, especially in view of item (i). It seems hopeless to conduct simple experiments (for instance, collisions between pairs of balls, or collisions between the block and the 
ground at one corner) in order to obtain values for the entries of $\mathcal{E}_{n}$. Is there another way to estimate them, except by adapting them to the experimental data obtained from each experimental test?

(iv) In both the block and the chain of balls examples, it appears that the subspace of admissible post-impact velocities can be spanned only if off-diagonal entries are introduced in the normal restitution matrix $\mathcal{E}_{n}$. These off-diagonal entries model distance effects between the various contact/impact points. Is this a general feature for multiple impacts in multibody systems?

The answers to such questions deserves future investigations. Some very preliminary answers may exist, however. For instance, it is known that if one contact in a chain of balls is very dissipative, then this contact acts as a "barrier" for the wave traveling through the chain $[23,42,43]$. Then the balls after the "barrier" do not undergo any shock and remain (in the initial configuration of Sect. 7.1), stuck after the collision. If the very dissipative contact is the first one, then Moreau's law, that is, $\mathcal{E}_{n}=\operatorname{diag}(e)$, may be adopted. To go further requires much more results on wave propagation into chains. One should also be reminded that the great advantage of these impact laws is their ease of use, as they involve very simple calculations to obtain the post-impact velocity (a matrix multiplied by a vector). This is very important in applications where the computational time is crucial. Whether there is a way to go beyond event-driven methods is another issue.

\section{Conclusions}

This paper examines the capabilities of a generalized kinematic restitution law, designed from a particular representation of the dynamics, when applied to the planar rocking block. It is shown that the energetic, kinematic, and kinetic physical constraints that have to be respected by the impact law, imply a set of restrictions on the post-impact motion and the restitution coefficients, depending on the block's aspect ratio (equivalently its kinetic angle). The fundamental issue is that the rocking motion involves some kinematic constraints (some contact points are fixed in the normal or/and the tangential directions at the contact points), and the proposed kinematic impact law adds other kinematic constraints. One of the conclusions of this work is that the widely used (in the earthquake engineering literature) angular velocity restitution coefficient has the interpretation of a tangential restitution and has therefore no reason to be a signed parameter. When applying such impact laws one, therefore, has to be careful not to violate the basic physical constraints by suitably choosing the coefficients within some intervals. The case of a chain of four balls is also treated, and illustrates further that the proposed kinematic law can be applied to various multibody systems undergoing multiple impacts. Whether or not such kinematic laws satisfy the desired properties for a multiple impact law as listed for instance in [31] is another issue, and not tackled in this paper.

Acknowledgements This work was performed with the support of the NSFC/ANR project Multiple Impact, ANR-08-BLAN-0321-01. H. Zhang was funded by China Scholarship Council No. 2009601276 and by ANR project Multiple Impact ANR-08-BLAN-0321-01.

\section{References}

1. Acary, V., Brogliato, B.: Numerical Simulation for Nonsmooth Dynamical Systems. Lecture Notes in Applied and Computational Mechanics, vol. 35. Springer, Heidelberg (2008) 
2. Andreaus, U., Casini, P.: On the rocking-uplifting motion of a rigid block in free and forced motion: influence of sliding and bouncing. Acta Mech. 138, 219-241 (1999)

3. Ballard, P.: The dynamics of discrete mechanical systems with perfect unilateral constraints. Arch. Ration. Mech. Appl. 154, 199-274 (2000)

4. Bernstein, D.S.: Matrix, Mathematics. Theory, Facts, and Formulas with Application to Linear Systems Theory. Princeton University Press, Princeton (2005)

5. Bowling, A., Flickinger, D.M., Harmeyer, S.: Energetically consistent simulation of simultaneous impacts and contacts in multibody systems with friction. Multibody Syst. Dyn. 22, 27-45 (2009)

6. Brach, R.M.: Mechanical Impact Dynamics. Wiley, New York (1991)

7. Brach, R.M.: Impact coefficients and tangential impacts. J. Appl. Mech. 64, 1014-1017 (1997)

8. Brogliato, B.: Nonsmooth Mechanics, 2nd edn. Springer, London (1999)

9. Brogliato, B.: Nonsmooth Impact Mechanics. LNCIS, vol. 220. Springer, London (1996)

10. Chatterjee, A., Ruina, A.: A new algebraic rigid-body collision law based on impulse space considerations. J. Appl. Mech. 65, 939-951 (1998)

11. Choi, J., Ryu, H.S., Kim, C.W., Choi, J.H.: An efficient and robust contact algorithm for a compliant contact force model between bodies of complex geometries. Multibody Syst. Dyn. 23, 99-120 (2010)

12. Dimitrakopoulos, E.G.: Analysis of a frictional oblique impact observed in skew bridges. Nonlinear Dyn. 60, 575-595 (2010)

13. Djerassi, S.: Collision with friction; Part A: Newton's hypothesis. Multibody Syst. Dyn. 29, 37-54 (2009)

14. Dzonou, R., Monteiro-Marques, M.D.P.: A sweeping process approach to inelastic contact problems with general inertia operators. Eur. J. Mech. A, Solids 26(3), 474-490 (2007)

15. Fielder, W.T., Virgin, L.N., Plaut, R.H.: Experiments and simulation of overturning of an asymmetric rocking block on an oscillating foundation. Eur. J. Mech. A, Solids 16(5), 905-923 (1997)

16. Flickinger, D.M., Bowling, A.: Simultaneous oblique impacts and contacts in multibody systems with friction. Multibody Syst. Dyn. 23, 249-261 (2010)

17. Flores, P., Leine, R., Glocker, C.: Modeling and analysis of planar rigid multibody systems with translational clearance joints based on the non-smooth dynamics approach. Multibody Syst. Dyn. 23, 165-190 (2010)

18. Förg, M., Pfeiffer, F., Ulbrich, H.: Simulation of unilateral constrained systems with many bodies. Multibody Syst. Dyn. 14, 137-154 (2005)

19. Frémond, M.: Collisions. Istituto Poligrafico e Zecca Dello Stato s.p.a., Roma (2007)

20. Frémond, M.: Rigid bodies collisions. Phys. Lett. A 204, 33-41 (1995)

21. Gale, D.: An indeterminate problem in classical mechanics. Am. Math. Mon. 59(5), 291-295 (1952)

22. Glocker, Ch.: Concepts for modeling impacts without friction. Acta Mech. 168, 1-19 (2004)

23. Glocker, C.: An introduction to impacts. In: Haslinger, J., Stavroulakis, G. (eds.) Nonsmooth Mechanics of Solids. CISM Courses and Lectures, vol. 485, pp. 45-102. Springer, Wien (2006)

24. Glocker, C., Aeberhard, U.: The geometry of Newton's cradle. In: Alart, P., Maisonneuve, O., Rockafellar, R.T. (eds.) Nonsmooth Mechanics and Analysis. Theoretical and Numerical Advances. AMMA, vol. 12, pp. 185-194. Springer, Berlin (2006)

25. He, K., Dong, S., Zhou, Z.: Multigrid contact detection method. Phys. Rev. E 75, 036710 (2007)

26. Heidenreich, B.: Small and half-scale experimental studies of rockfall impacts on sandy slopes. PhD thesis, Ecole Polytechnique Fédérale de Lausanne, section de Génie Civil (CH), Thèse no 3059 (2004)

27. Housner, G.W.: The behaviour of inverted pendulum structures during earthquakes. Bull. Seismol. Soc. Am. 53(2), 403-417 (1963)

28. Lancaster, P., Tismenetsky, M.: The Theory of Matrices, 2nd edn. Academic Press, San Diego (1985)

29. Leine, R.I., van de Wouw, N.: Stability properties of equilibrium sets of non-linear mechanical systems with dry friction and impact. Nonlinear Dyn. 51, 551-583 (2008)

30. Lipscombe, P.R., Pellegrino, S.: Free rocking of prismatic blocks. J. Eng. Mech. 119(7), 1387-1410 (1993)

31. Liu, C., Zhao, Z., Brogliato, B.: Frictionless multiple impacts in multibody systems: Part I. Theoretical framework. Proc. R. Soc. A, Math. Phys. Eng. Sci. 464(2100), 3193-3211 (2008)

32. Liu, C., Zhao, Z., Brogliato, B.: Energy dissipation and dispersion effects in a granular media. Phys. Rev. E 78(3), 031307 (2008)

33. Liu, C., Zhao, Z., Brogliato, B.: Variable structure dynamics in a bouncing dimer. INRIA Research Report 6718, November 2008. http://hal.inria.fr/inria-00337482/fr/

34. Liu, C., Zhao, Z., Brogliato, B.: Frictionless multiple impacts in multibody systems: Part II. Numerical algorithm and simulation results. Proc. R. Soc. A, Math. Phys. Eng. Sci. 465(2101), 1-23 (2009)

35. Lun, C.K.K., Bent, A.A.: Computer simulation of simple shear flow of inelastic, frictional spheres. In: Thortin (ed.) Powders and Grains, vol. 93, pp. 301-306 (1993)

36. Milne, J.: Seismic experiments. Trans. Seism. Soc. Jpn. 8, 1-82 (1885) 
37. Modarres Najafabadi, S.A., Kövecses, J., Angeles, J.: Generalization of the energetic coefficient of restitution for contacts in multibody systems. J. Comput. Nonlinear Dyn. 3, 041008 (2008)

38. Modarres Najafabadi, S.A., Kövecses, J., Angeles, J.: Impacts in multibody systems: modeling and experiments. Multibody Syst. Dyn. 20, 163-176 (2008)

39. Monteiro-Marques, M.D.P.: Differential Inclusions in Nonsmooth Mechanical Problems. Shocks and Dry Friction. Progress in Nonlinear Differential Equations and their Applications, vol. 9. Birkhauser, Basel (1993)

40. Moreau, J.J.: Some numerical methods in multibody dynamics: application to granular materials. Eur. J. Mech. A, Solids 13(4), 93-114 (1994)

41. Paoli, L.: Continuous dependence on data for vibro-impact problems. Math. Models Methods Appl. Sci. 15(1), 1-41 (2005)

42. Payr, M., Glocker, C., Bösch, C.: Experimental treatment of multiple contact collisions. In: Euromech Conference ENOC, Eindhoven, 7-12 August, pp. 450-459 (2005)

43. Payr, M.: An experimental and theoretical study of perfect multiple contact collisions in linear chains of bodies. PhD thesis ETH Zurich, No. 17808, ETH E-Collection, 2008, 171 pages. Available at http://www.zfm.ethz.ch/e/dynamics/payr_collisions.htm

44. Payr, M., Glocker, C.: Oblique frictional impact of a bar: analysis and comparison of different impact laws. Nonlinear Dyn. 41, 361-383 (2005)

45. Pena, F., Prieto, F., Lourenço, P.B., Campos Costa, A., Lemos, J.V.: On the dynamics of rocking motion of single rigid-block structures. Earthquake Eng. Struct. Dyn. 36, 2383-2399 (2007)

46. Perry, J.: Note on the rocking of a column. Trans. Seism. Soc. Jpn. 3, 103-106 (1881)

47. Pfeiffer, F., Glocker, C.: Multibody Dynamics with Unilateral Contacts. Wiley Series in Nonlinear Science (1996)

48. Prieto, F., Lourenço, P.B.: On the rocking behavior of rigid objects. Meccanica 40, 121-133 (2005)

49. Stewart, D.E., Trinkle, J.C.: An implicit time-stepping scheme for rigid body dynamics with inelastic collisions and Coulomb friction. Int. J. Numer. Methods Eng. 39, 2673-2691 (1996)

50. Stronge, W.J.: Impact Mechanics. Cambridge University Press, Cambridge (2000)

51. Stronge, W.J., James, R., Ravani, B.: Oblique impact with friction and tangential compliance. Philos. Trans. R. Soc. Lond. A 359, 1-19 (2001)

52. Taniguchi, T.: Non-linear response analyses of rectangular rigid bodies subjected to horizontal and vertical ground motion. Earthquake Eng. Struct. Dyn. 31, 1481-1500 (2002)

53. Walton, O.R.: Numerical simulation of inelastic, frictional particle-particle interactions. In: Roco, M.C. (ed.) Particulate Two-Phase Flow. Butterworth/Heinemann, Stoneham (1992)

54. Yilmaz, C., Gharib, M., Hurmuzlu, Y.: Solving frictionless rocking block problem with multiple impacts. Proc. R. Soc. A, Math. Phys. Eng. Sci. 465, 3323-3339 (2009)

55. Yim, C.S., Chopra, A.K., Penzien, J.: Rocking response of rigid blocks to earthquakes. Earthquake Eng. Struct. Dyn. 8(6), 565-587 (1980)

56. Zhang, H., Brogliato, B., Liu, C.: Study of the planar rocking-block dynamics without and with friction: critical kinetic angles. Submitted

57. Zhang, H., Brogliato, B.: The planar rocking block: analysis of kinematic restitution laws, and a new rigid-body impact model with friction. INRIA Research Report RR-7580, March 2011. http://hal.inria.fr/inria-00579231/en/

58. Zhao, Z., Liu, C., Brogliato, B.: Planar dynamics of a rigid body system with frictional impacts. II. Qualitative analysis and numerical simulations. Proc. R. Soc. A, Math. Phys. Eng. Sci. 465(2107), 2267$2292(2009)$ 